\title{
Pattern Recognition Receptors in Innate Immunity to Obligate Intracellular Bacteria
}

\author{
James R. Fisher ${ }^{1,2}$, Zachary D. Chroust ${ }^{2}$, Florence Onyoni ${ }^{3}$ and Lynn Soong ${ }^{1,4,5, *}$
}

\begin{abstract}
Host pattern recognition receptors (PRRs) are crucial for sensing pathogenic microorganisms, initiating innate responses, and modulating pathogenspecific adaptive immunity during infection. Rickettsia spp., Orientia tsutsugamushi, Anaplasma spp., Ehrlichia spp., and Coxiella burnetii are obligate intracellular bacteria that can replicate only within host cells and must evade immune detection to successfully propagate. These five bacterial species are zoonotic pathogens of clinical or agricultural importance, yet uncovering how immune recognition occurs has remained challenging. Recent evidence from in vitro studies and animal models has yielded new insights into the types and kinetics of PRR activation during infection with Rickettsia spp., A. phagocytophilum, E. chaffeensis, and C. burnetii. However, much less was known about PRR activation in O. tsutsugamushi infection until the recent discovery of the role of the C-type lectin receptor Mincle during lethal infection in mice and in primary macrophage cultures. This review provides a brief summary of the clinical and epidemiologic features of these five bacterial infections, with a focus on the fundamental biologic facets of infection, and recent advances in host recognition. In addition, knowledge gaps regarding the innate recognition of these bacteria in the context of disease pathogenesis are discussed.
\end{abstract}

Key words: pattern recognition receptor, innate immunity, obligate intracellular bacteria, Orientia tsutsugamushi, Rickettsia, Anaplasma, Ehrlichia, Coxiella burnetii
Edited by:

Ruifu Yang, Beijing Institute of

Microbiology and Epidemiology

Reviewed by:

Xiaolu Xiong, Institute of

Microbiology and Epidemiology,

Academy of Military Medical Sciences

Another reviewer chose to be

anonymous

*Corresponding author:

E-mail: lysoong@utmb.edu

${ }^{1}$ Department of Microbiology and Immunology, University of Texas Medical Branch, Galveston, TX, USA

${ }^{2}$ School of Medicine, University of

Texas Medical Branch, Galveston,

TX, USA

${ }^{3}$ Graduate School of Biomedical

Sciences, University of Texas Medical Branch, Galveston, TX, USA

${ }^{4}$ Department of Pathology, University of Texas Medical Branch, Galveston, TX, USA

${ }^{5}$ Institute for Human Infections and Immunity, University of Texas Medical Branch, Galveston, TX, USA

Received: August 272021 Revised: October 022021

Accepted: October 082021

Published Online: October 252021

\section{BACKGROUND}

Pattern recognition receptors (PRRs) sense a broad array of self- or non-self-molecules, thus serving as sentinels of infection. They detect pathogen-associated molecular patterns (PAMPs) and host damage-associated molecular patterns (DAMPs), and subsequently initiate immune responses [1-5]. Four major families of PRRs have been identified, each sensing distinct molecular motifs or structures, and playing specific or cooperative roles during infection. The cross-talk among different or identical receptor family members, as well as their downstream signaling pathways, can lead to pathogen and host context-dependent immunological outcomes [2-5]. Different PRRs collectively influence both the innate and adaptive arms of immunity, thus resulting in pathogen-specific responses, which can lead to infection control or promote disease pathogenesis. 
Toll-Like Receptors (TLRs), the first identified family of PRRs [6], are transmembrane proteins in both plasma and endosomal membranes [2]. TLRs located on the cell surface detect bacterial components, including lipoproteins (TLRs 1, 2, and 6) [7-10], lipopolysaccharide (TLR4) [11], and flagellin (TLR5) [12]. In contrast, endosomal TLRs detect nucleic acids of viral or parasitic origin, including double-stranded RNA (TLR3) [13], single-stranded RNA (TLR7 and TLR8) [14-17], and CpG-containing single-stranded DNA (TLR9) [18]. After ligand binding, TLRs interact with an adaptor complex consisting of either 1) myeloid differentiation primary-response protein 88 (MyD88), which is shared by all TLRs, or 2) Toll/IL-1Rdomain-containing adapter-inducing interferon- $\beta$ (TRIF), which is used by TLR3 and TLR4 [2]. The result of TLR signaling via MyD88 is the transcription of NF- $\mathrm{KB}-$ and AP-1-dependent genes, whereas signaling via TRIF results in transcription of NF- $\mathrm{KB}, \mathrm{AP}-1$, and IRF3 controlled genes and the induction of necroptosis [2]. Although TLRs have also been implicated in sensing DAMPs, this aspect of signaling is beyond the scope of this article and has been extensively reviewed elsewhere [19,20].

Nucleotide Binding Oligomerization Domain-Like Receptors (NLRs) are located in the cytoplasm and contain a nucleotide-binding domain and a leucine-rich repeat domain, which is involved in sensing PAMPs and DAMPs [3]. NLRs are grouped into subfamilies according to the presence of additional domains, including caspase activation and recruitment domains (NLRC) and pyrin domains (NLRP) [3]. Within the NLRC subfamily, NOD1 and NOD2 have been widely studied and are known to recognize the building blocks of peptidoglycan (muropeptides and muramyl dipeptides, respectively) [21,22]. Activated NOD1 and NOD2 interact with the receptor-interactingserine-threonine-kinase 2 (RIP2) and subsequently stimulate NF- $\mathrm{KB}$ mediated transcription or alternatively IRF7/3, thereby inducing interferon responses [3]. NLRC4, another member of the NLRC subfamily, recognizes intracellular flagellin and contributes to inflammasome assembly, thus leading to Caspase- 1 activation and secretion of the proinflammatory cytokine IL-1 $\beta$ [3,23-26]. Members of the NLRP subfamily, including NLRP3, respond to potassium efflux, reactive oxygen species, and bacterial lipoproteins, and subsequently activate inflammasomes and the secretion of proinflammatory cytokines (namely IL-1 $\beta$ ) [3]. NLRs have also been implicated in numerous other cellular processes, including autophagy, and have been shown to engage in crosstalk with TLRs and RIG-I (described below) [3].

Like NLRs, Retinoic Acid-Inducible Gene I (RIG-I)Like Receptors (RLRs) are localized in the cytosol [27]. The RLR family includes RIG-I, melanoma differentiationassociated protein 5 , and laboratory of genetics and physiology protein 2 [4]. Family members contain a central helicase domain and carboxy-terminal domain, which collectively recognize immunostimulatory RNA bearing 5'-PPP moieties [4].After activation, RIG-I interacts with mitochondrial antiviral-signaling protein and initiates type-1 interferon responses and NF- $\mathrm{KB}$ translocation [4]. Although activation of RLRs has historically been implicated in sensing viral infection, recent evidence has shown that RLRs may also sense mitochondrial RNA [28,29] and play a wider role in sensing DAMPs than previously understood.

C-Type Lectin Receptors (CLRs) are a diverse superfamily comprising more than 1,000 proteins that, by definition, contain at least one C-type lectin-like domain [5]. CLRs are expressed predominantly in myeloid cells and are secreted or anchored to the plasma membrane $[5,30]$. This family of receptors recognizes endogenous and exogenous carbohydrate or glycolipid moieties [5]. Activation of CLRs influences inflammation through the adaptor protein spleen tyrosine kinase (SYK). CLRs interact with SYK via an immunoreceptor tyrosine-based inhibitory or immunoreceptor tyrosine-based activation motif in its own cytoplasmic tail, or through coupling with signaling partners (mainly Fc $\gamma$ Rs or DAP10/12) [5]. Most CLRs studied in the context of bacterial recognition are members of the Group II asialoglycoprotein receptor family, including Dectin-1 and dendritic cell immunoreceptor subfamilies [5]. Mincle (Macrophage inducible $\underline{\mathrm{C}}$-type lectin; also known as Clec4e), the best-characterized CLR, is a member of the dendritic cell immunoreceptor subfamily known to recognize bacterial glycolipids as well as host DAMPs [5]. The outcomes of Mincle activation are highly varied and context dependent, and include inflammatory macrophage $(M \Phi)$ polarization, induction of type 1-skewed $\mathrm{T}$ helper responses, and proinflammatory cytokine production [5].

Studies identifying PRR activation during bacterial infection have focused extensively on extracellular or facultative intracellular bacteria. However, scant evidence is available for obligate intracellular bacteria, a group of clinically important organisms that are highly prevalent worldwide [31,32]. This unique group includes the Rickettsiales (Rickettsia spp., O. tsutsugamushi, Anaplasma spp., and Ehrlichia spp.) as well as C. burnetii. Virtually all these obligate intracellular bacteria have zoonotic infection cycles (Table 1). For example, Rickettsia spp. is found on all continents except Antarctica and is transmitted to humans via numerous blood-feeding arthropods (including ticks, lice, and fleas) [32,33], whereas O. tsutsugamushi is endemic across Southeast Asia and is transmitted via mites [34]. In contrast, Anaplasma and Ehrlichia are predominantly found in the United States and are transmitted via numerous tick species $[35,36]$. C. burnetii, although found globally, is endemic to the Mediterranean region and is commonly spread via livestock secretions [37]. However, recent evidence indicates that ticks may serve as a vector of transmission [38-40].

The biology of obligate intracellular pathogens necessitates immune evasion, because replication can occur only within host cells. Thus, these bacteria, in contrast to extracellular bacteria, exhibit many unique characteristics, most prominently extensive genome reduction and the lack of immunostimulatory cell wall components and extensive genome reduction [31]. O. tsutsugamushi, for example, lacks 


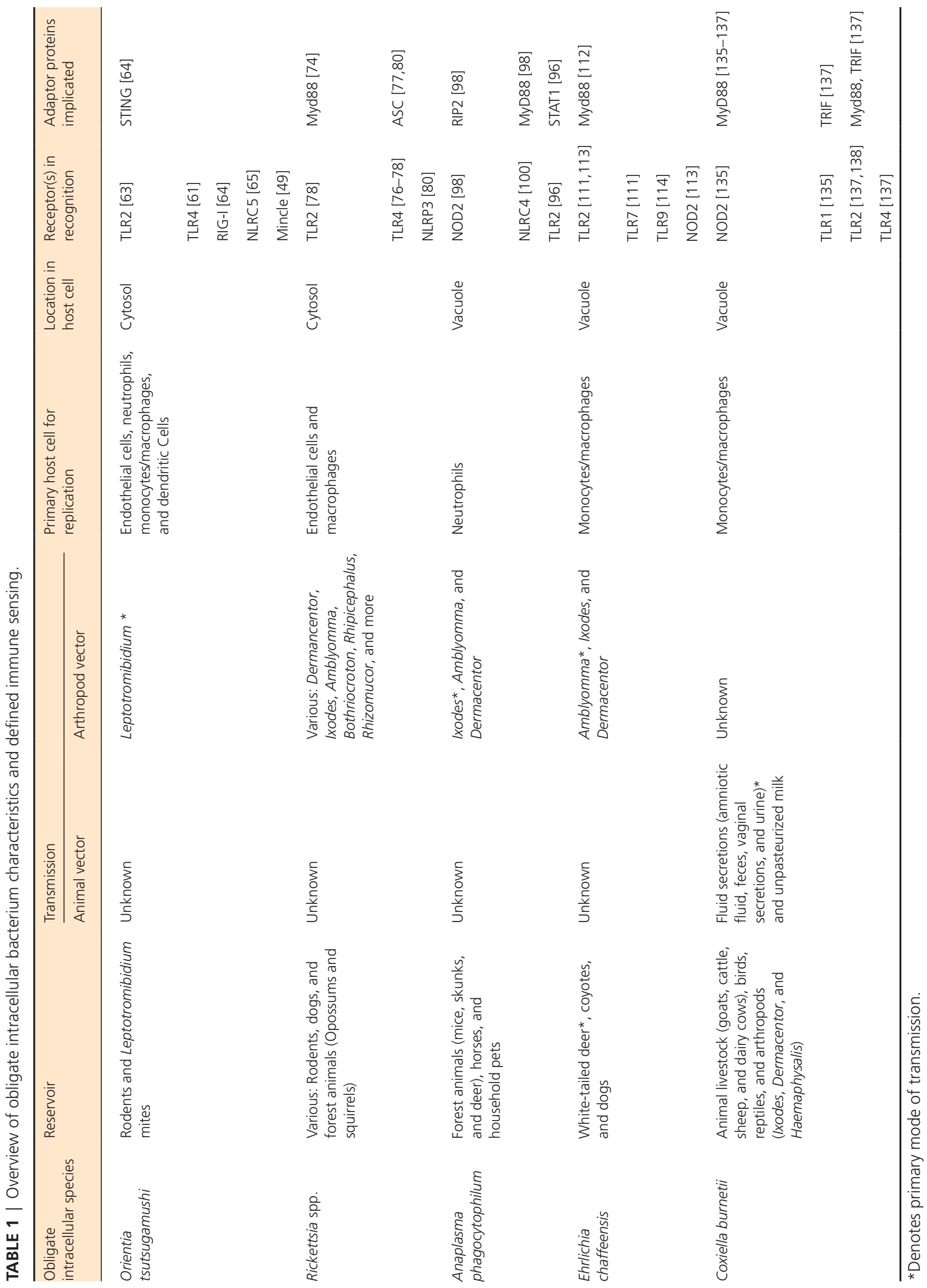


biosynthetic pathways for both peptidoglycan and lipopolysaccharide (LPS) [41]. Additionally, the genome of O. tsutsugamushi comprises $\sim 1.2$ million base pairs [41], in stark contrast to the $\sim 5$ million base pair genome of Salmonella typhi [42] and the $\sim 4.5$ million base pair genome of Escherichia coli [43].Very little is known regarding the recognition of obligate intracellular bacteria and how PRRs orchestrate the immune response to these pathogens. Direct comparative analyses among these bacteria are severely lacking. To our knowledge, only one report has used primary human dendritic cells and directly compared innate immune signatures among obligate intracellular bacterial species including $O$. tsutsugamushi and C. burnetii [44]. Studies aimed at defining the PRR response have been complicated by the challenges of working with these agents, including technical difficulties in propagating large-scale cultures and the necessity for biocontainment facilities for O. tsutsugamushi, Rickettsia spp., and C. burnetii $[31,41,45]$.

Although highly treatable, the Rickettsiales and C. burnetii are often overlooked causes infection, and severe disease usually includes immunopathogenic features [32,46-48]. Thus, thoroughly defining activated PRRs and their effects on immune signatures would yield substantial insights into disease pathogenesis and potential treatments for severely ill people. In this review, we discuss critical clinical and epidemiologic features of the Rickettsiales and C. burnetii, along with recent advances in the understanding of PRR sensing during initial infections with these obligate intracellular bacteria. Finally, we highlight key areas for future studies to define the potential links between PRRs and disease pathogenesis.

\section{METHODS}

\section{Databases, search strategy, study selection, and RNA sequencing}

Articles on the obligate intracellular bacteria of interest were identified through searching of relevant publications from electronic sources. The searching was performed via Ovid-Medline and PubMed-Medline. Studies were identified by combining search terms for the bacteria of interest and PRR of interest. For example, studies for O. tsutsugamushi were identified by searching for "Orientia and TLR," "Orientia and NLR," "Orientia and RLR," and "Orientia and CLR." JRF and ZDC reviewed abstracts generated by the search for relevance and, unless the source was a seminal publication, included only reports from the past 10 years. Host gene transcriptional profiles were based on late stages of lethal infection in mice, and tissue analyses using NanoString, RNAseq, or qRT-PCR approaches $[49,50]$.

\section{ORIENTIA TSUTSUGAMUSHI}

\section{Epidemiology and clinical features}

O. tsutsugamushi is the causative agent of scrub typhus, a life-threatening disease with increasing impact globally. Approximately 1 million cases of scrub typhus occur each year in an endemic region termed the "tsutsugamushi triangle," which spans southeast Asia and northern Australia [34]. However, recent reports have indicated the serological prevalence of scrub typhus in historically non-endemic regions, including South America [51,52] and Africa $[53,54]$. Although rodents may serve as an animal reservoir for this bacterium [55], O. tsutsugamushi is predominantly maintained in Leptotrombidium mites (commonly known as chiggers) [56]. The bacterium is transmitted to humans via bites by larval stage mites, and disease pathogenesis occurs mainly in highly vascularized organs (such as the lung, liver, or brain) [34]. Scrub typhus may manifest as interstitial pneumonia, liver damage, and meningoencephalitis [34]. If untreated, the disease can progress to multi-organ failure and is associated with fatality rates ranging from $0 \%$ to $70 \%$ (median of $6 \%$ ) $[34,57,58]$.

\section{TLR/RIG-I/NLR-mediated immune recognition}

O. tsutsugamushi is an LPS-negative, Gram-negative coccobacillus that infects primarily endothelial cells and phagocytes (MFs, neutrophils, and dendritic cells) [41]. Compared with the other four bacterial species of interest, O. tsutsugamushi has a unique biology as well as host recognition mechanisms (Fig 1). After the bacterium is internalized via endocytosis or phagocytosis, it rapidly escapes the endosome and freely inhabits the cytosol [41]. The bacteria can use microtubules to traffic to the perinuclear region where replication occurs. O. tsutsugamushi replicates slowly, with peak rates occurring over 1-5 days post-infection (dpi), and then exits host cells via a budding-like mechanism $[41,59]$. A recent report has shown that O. tsutsugamush $i$ actively inhibits NF- $\kappa \mathrm{B}$ activation and consequently evades host responses during its replication process [60]. However, few reports have examined the innate recognition of O. tsutsugamushi, and the mechanisms of PRR sensing remain obscure.

Although TLR activation during O. tsutsugamushi infection has been demonstrated, the evidence remains debatable. One study in humans has suggested that a TLR4 mutation (D299G) is associated with increased scrub typhus susceptibility [61]. This mutation affects the ligand binding pocket of TLR 4 and has been implicated in susceptibility to tuberculosis [62]. Because O. tsutsugamushi lacks LPS, the observed link between TLR 4 and susceptibility to scrub typhus is likely to be due to yet-undefined or indirect mechanisms. This possibility has been supported by another study in human TLR4- or TLR2-transfected HEK293 cells, which has shown that TLR 4 does not directly recognize O. tsutsugamushi, whereas TLR2 may sense bacterial components and promote IL-6 secretion [63]. The role of TLR2 in O. tsutsugamushi infection is supported in bone marrow-derived dendritic cells (BMDCs): infected TLR $2^{-/-}$BMDCs have been found to secrete less IL- 6 and TNF $\alpha$ than wild-type (WT) controls [63]. Intradermal infection of TLR $2^{-/-}$and WT C57BL/6 mice (producing a self-limiting infection) has not been observed to result in differences in survival or bacterial loads [63]. However, after intraperitoneal infection (producing lethal infection), TLR $2^{-/-}$mice have milder disease scores and pathology, but greater bacterial loads in the 


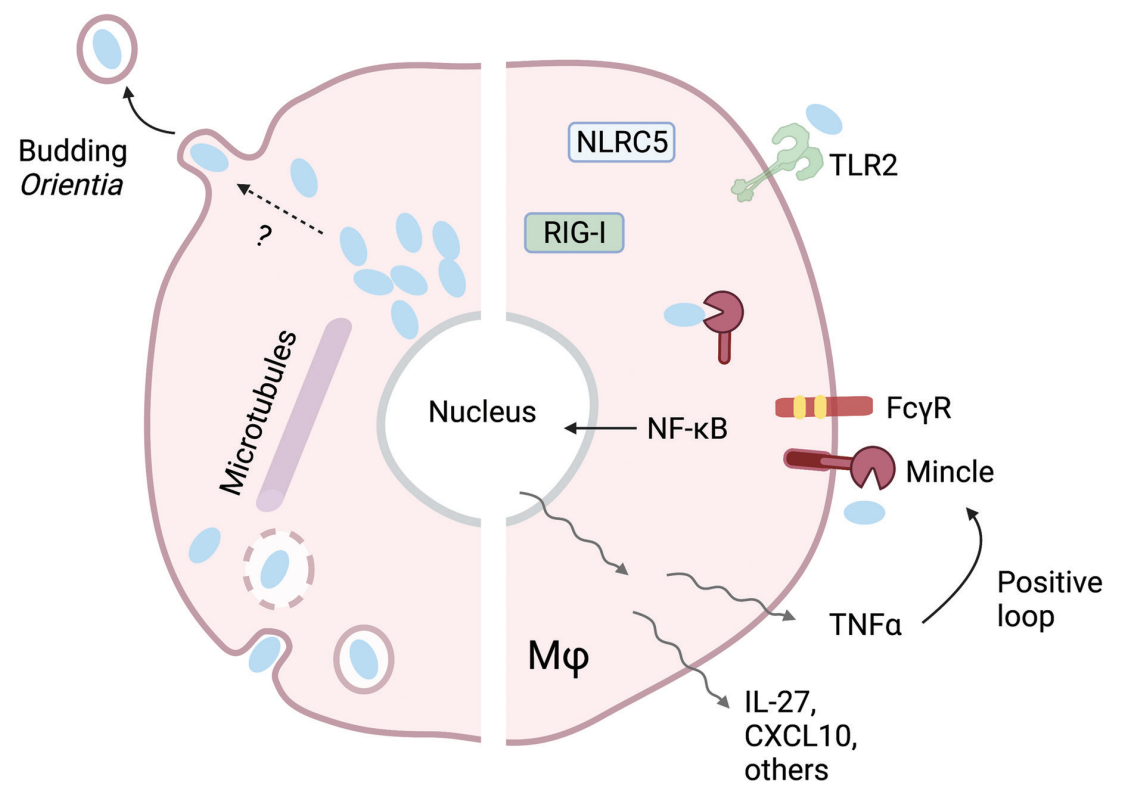

FIGURE 1 | Orientia tsutsugamushi intracellular life cycle and host innate responses.

After entering the host cell through endocytosis, Orientia bacteria rapidly escape the endosome, move to the perinuclear region via microtubules, and replicate freely (and slowly) in the cytoplasm. O. tsutsugamushi then exits the cell via a poorly defined budding mechanism. Host immune recognition is mediated by Mincle/Fcy receptor-regulated mechanisms, although the involvement of other sensors (TLR2, RIG-I, and NLRC5) has been reported. Mincle signaling promotes expression of NF-KB-induced proinflammatory genes, including TNF $\alpha$, IL-27, and CXCL10. TNF $\alpha$ in turn promotes Mincle expression via a positive feedback loop, thus enhancing proinflammatory responses.

lung, spleen, and peritoneum, than WT mice. Because O. tsutsugamushi-infected TLR2 $2^{-/-}$and WT mice have comparable levels of IL- 6 and TNF $\alpha$ transcripts, the biological function of TLR 2 in this infection is unclear.

Like the roles of TLRs, the role of the cytosolic sensor RIG-I in sensing O. tsutsugamushi is also under debate. Min et al. have shown that infected mouse embryonic fibroblasts lacking functional MAVS, RIG-I, or STING express fewer IFN- $\beta$ and TNF $\alpha$ transcripts than their WT counterparts early in infection [64]. However, data for other in vitro time points or in mice are lacking.

Controversial evidence of NLR activation during O. tsutsugamushi infection has also been reported. In in vitro studies in nonprofessional phagocytes ( $\mathrm{HeLa}$ and primary human aortic endothelial cells), O. tsutsugamushi has been found to decrease NLRC5 protein levels in late infection, at 72 hours post-infection (hpi), thereby downregulating major histocompatibility complex-1 expression [65]. However, in THP-1 monocyte-like cells, NLRC5 expression has been found to only temporarily decrease early in infection and to rebound by 72 hpi [65]. Additionally, Cho et al. have reported infection-associated NOD1 protein expression, as well as reduced proinflammatory cytokine protein expression in NOD1-knock-down cells [66]. However, the involvement of NOD1/NOD2 in O. tsutsugamushi infection has not been validated by a separate team in mouse BMMФs lacking RIP2, NLRP3, NLRP4, or AIM2 [67].

\section{CLR-mediated immune recognition}

The first evidence of the role of Mincle, a unique member of the CLR family, in response to O. tsutsugamushi was reported in 2021, as identified through comprehensive molecular and immunological approaches [49]. First, several host gene expression profiling analyses of tissues collected from lethally infected C57BL/6 mice (via the intravenous route) revealed a 36-fold increase in Mincle (also known as Clec4e) in the lungs, as well as an $\sim 400-14,000$-fold increase in the brains at $10 \mathrm{dpi}$ (before host death) (Table 2). Simultaneously, a low degree of TLR, NLR, and RLR expression was observed. Second, multiple approaches (NanoString, qRT-PCR, western blotting, and immunofluorescence staining) consistently confirmed Mincle activation in conjunction with the upregulation of Mincle signaling partners (Fc $\gamma \mathrm{Rs}$ ) and proinflammatory cytokines/chemokines (CXCL9-11, TNFo, IL-27) in inflamed or damaged lungs. Third, our in vitro studies in BMM $\Phi_{\text {s revealed upreg- }}$ ulated Mincle RNA and protein levels in response to live or inactivated O. tsutsugamushi, both of which positively correlated with upregulated type 1-promoting markers (CXCL9-11, TNF $\alpha$, and IL-27), M $\Phi$ chemotactic markers (CCL2-7), and the neutrophil chemotactic marker CXCL1. In contrast, infected $\mathrm{Mincle}^{-/-}$BMMФs exhibited abrogated transcription of CCL2 and CXCL1, thus implicating the receptor in propagating inflammation. Finally, given that both bacterium-carrying and uninfected $M \Phi_{\mathrm{s}}$ express Mincle, and that tissue Mincle levels peak at late stages of disease, we speculated that host factors contribute to driving Mincle expression. Indeed, we have confirmed a positive, synergetic role of TNF $\alpha$ in regulating Mincle expression.

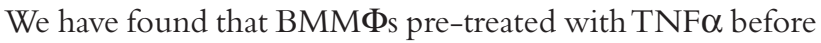
infection greatly enhance Mincle, IL-27, and CXCL10 expression, whereas some of these effects are markedly 
TABLE 2 | PRR gene expression in O. tsutsugamushi-infected C57BL6 mice.

\begin{tabular}{|c|c|c|c|c|}
\hline & \multirow[b]{2}{*}{ Gene/alias (encoded protein) } & \multicolumn{3}{|c|}{ Fold change (D10 vs. D0) } \\
\hline & & $\begin{array}{l}\text { Lung NanoString } \\
\text { (Ref. [49]) }\end{array}$ & $\begin{array}{l}\text { Brain NanoString } \\
\text { (Ref. [49]) }\end{array}$ & Brain RNAseq \\
\hline \multirow[t]{3}{*}{ CLR } & $\begin{array}{l}\text { Clec4e (Macrophage Inducible C-type Lectin; } \\
\text { Mincle) }\end{array}$ & 36.00 & 441.21 & 14082.01 \\
\hline & Clec4d (Macrophage C-type Lectin; MCL) & - & - & 118.47 \\
\hline & Clec5a (Myeloid DAP12-Associating Lectin 1; MDL) & 6.96 & 6.34 & 4.15 \\
\hline \multirow[t]{4}{*}{ CLR Partner } & Fcgr4 (Fcy Receptor 4) & 18.77 & 517.89 & 138.43 \\
\hline & Fcgr1 (Fcy Receptor 1) & 7.11 & 12.79 & 7.21 \\
\hline & Fcgr3 (Fcy Receptor 3) & 3.81 & 7.93 & 5.54 \\
\hline & Fcgr2b (Fcy Receptor 2b) & 3.20 & 14.81 & 5.37 \\
\hline \multirow[t]{5}{*}{ TLR } & Toll-Like receptor 1 & 3.13 & 9.19 & 9.88 \\
\hline & TIr2 & 1.97 & ns & 8.46 \\
\hline & TIr4 & 0.98 & 5.46 & 4.64 \\
\hline & TIr6 & 4.28 & - & 2.84 \\
\hline & MyD88 & 1.85 & 3.54 & 2.86 \\
\hline \multirow[t]{5}{*}{ NLR and RLR } & NIrc5 (NLR Family CARD Domain Containing 5) & - & - & 79.53 \\
\hline & $\begin{array}{l}\text { Nod1 (Nucleotide-Binding Oligomerization Domain- } \\
\text { Containing Protein 1) }\end{array}$ & 1.38 & - & 4.35 \\
\hline & $\begin{array}{l}\text { Nod2 (Nucleotide-Binding Oligomerization Domain- } \\
\text { Containing Protein 2) }\end{array}$ & 2.60 & ns & 4.48 \\
\hline & NIrp3 (NLR Family Pyrin Domain Containing 3) & 3.29 & - & 3.46 \\
\hline & Ddx58 (RIG-I, Retinoic Acid-Inducible Gene I) & 0.80 & 6.53 & 4.61 \\
\hline
\end{tabular}

All values presented are statistically significant changes $(p<0.05)$ unless denoted not significant (ns).

diminished in infected Mincle ${ }^{-/-}$cells. Together, our studies have provided the first confirmation of the important role of Mincle in sensing live versus inactivated O. tsutsugamushi. We have proposed that Mincle/FcgR activation via bacterial glycoprotein/glycolipid motifs and innate host factors (e.g., TNF $\alpha$ ) is a major mechanism that programs MFs to a M1-like phenotype, thus contributing to Th1/M1-skewed inflammatory responses in O. tsutsugamushi-infected mice and humans (Fig 1) [49,50,68-71].

\section{Knowledge gaps and future studies}

Evidence of PRR involvement during O. tsutsugamushi infection has been enigmatic. Research has focused on examining classical PRRs, such as TLRs, RIG-I, and NLRs; however, none of these receptors play significant roles during infection. The studies on TLR2/4 are inconclusive [63], and linking these receptors to the biology of O. tsutsugamushi is challenging, because this bacterium lacks LPS and conventional peptidoglycan. RIG-I- or NLR-related studies have intrinsic limitations, owing to the use of mouse embryonic fibroblasts or other cell lines $[64,66]$. Mincle-mediated pathways not only sense and differentiate live versus inactivated O. tsutsugamushi but also enhance the inflammatory responses in $M \Phi_{\mathrm{S}}$ [49], thus prompting many questions. Is Mincle a key sensor during infection in experimental animals and in humans? If so, what are the bacterium- and/ or host-derived ligands for Mincle activation at early versus late stages of infection? How does Mincle interact with other PPRs in infection control and/or immunopathogenesis? Studies aimed at assessing the biological functions of Mincle on in vivo infection should yield insights into the immune recognition of this bacterium, which has been severely neglected in prior studies.

\section{RICKETTSIA SPP.}

\section{Epidemiology and clinical features}

Rickettsia spp. are found on all continents except Antarctica, and cause a wide range of human diseases [33]. Bacteria in this genus are classified into four groups on the basis of taxonomy and associated epidemiologic features. The spotted fever group (SFG) includes $R$. rickettsii (Rocky Mountain spotted fever), $R$. conorii (Boutonneuse fever), $R$. africae (African tick-bite fever), and $R$. parkeri (maculatum disease), which are transmitted to humans via ticks. The transitional group includes $R$. akari (Rickettsialpox), R. australis (Queensland fever), and R. felis (flea borne spotted fever), 
which are transmitted to humans via fleas, ticks, or mites. The typhus group (TG) consists of R. prowazekii (epidemic typhus) and R. typhi (murine typhus), which are transmitted to humans via fleas, lice, or flying squirrels. Finally, the ancestral group is composed of $R$. canadensis and $R$. bellii, and is not associated with any human diseases [31,72]. Rickettsioses display a diverse array of clinical symptoms and severity. Most infections begin with constitutional symptoms accompanied by rash [32]. However, the disease can progress to multiorgan failure and other life-threatening syndromes if not promptly treated [32]. Case fatality rates differ greatly among rickettsioses, and those of Rocky Mountain spotted fever and epidemic typhus rank among the highest (15-65\%) [72,73]. Therefore, $R$. rickettsii and $R$. prowazekii have garnered substantial research interest because of their high infectivity and mortality, as well as their potential for use as bioterrorism agents [72]. Interestingly, although no fatal cases have been reported, recrudescence of epidemic typhus, known as Brill-Zinsser disease, can occur years after the initial infection [32].

\section{TLR/MyD88-mediated immune recognition}

Rickettsia are Gram-negative, LPS-positive bacilli that infect primarily host endothelial cells and $M \Phi_{\mathrm{s}}$ [72]. After entering a host cell via endocytosis, Rickettsia escape the endolysosome and subsequently replicate freely within the cytoplasm in a manner similar to that of O. tsutsugamushi [31]. The ability to subvert autophagy plays a major role in bacterial survival, but the underlying mechanisms remain largely unexplored $[74,75]$. Replication is followed by direct invasion of neighboring cells (spotted fever group) or host cell lysis (typhus group) (Fig 2), in sharp contrast to the budding mechanism used by O. tsutsugamushi [31].

TLR2/4- and MyD88-mediated mechanisms are the best-characterized pathways for innate recognition of Rickettsia. Early evidence has indicated that $\mathrm{C} 3 \mathrm{H} / \mathrm{HeJ}$ mice (which are naturally deficient in TLR 4 function) are more susceptible to $R$. conorii challenge and show greater bacterial loads in the brain and lungs than $\mathrm{C} 3 \mathrm{H} / \mathrm{HeN}$ mice (which are TLR 4 competent) [76]. Infected $\mathrm{C} 3 \mathrm{H} / \mathrm{HeJ}$ mice also display diminished splenic natural killer cell activation, but this effect is rescued by adoptive transfer of TLR 4 competent DCs, thus implicating TLR4 as a driver of this process [76]. Additionally, the secretion of proinflammatory cytokines (IL-1 $\alpha$ and TNF $\alpha$ ) is much lower in infected $\mathrm{C} 3 \mathrm{H} / \mathrm{HeJ}$ primary brain microvascular endothelial cells than $\mathrm{C} 3 \mathrm{H} / \mathrm{HeN}$ cells [76]. TLR 4 has also been shown to recognize $R$. australis [77]. Infected TLR $4^{-/}$C57BL/6 BMMФs produce fewer pro-IL-1 $\beta$ transcripts than WT controls and bear greater bacterial loads [77]. Additionally, WT but not TLR $4^{-/-}$BMM $\Phi$ s produce pro-IL-1 $\beta$ transcripts in response to purified $R$. australis LPS, thereby indicating that rickettsial LPS is the likely stimulus for TLR4. Thus, TLR 4 recognizes $R$. conorii and $R$. australis, and contributes to generating the proinflammatory response.

TLR2 also plays a role in sensing Rickettsia. QuevedoDiaz et al. have used in vitro systems to examine whether $R$. akari activates TLR2/4 [78]. The authors added heatkilled $R$. akari to HEK293T cells stably transfected to express human TLR2 or TLR4. After addition of heat-killed

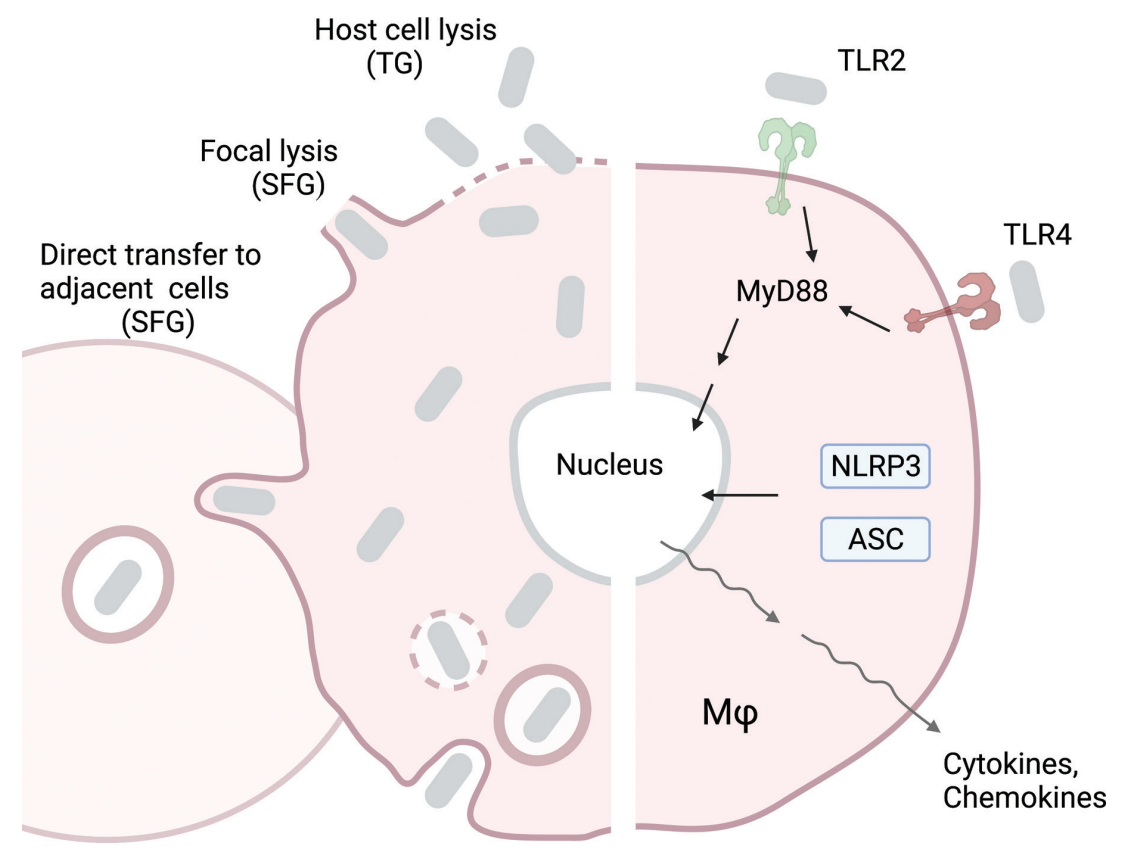

FIGURE 2 | Rickettsia spp. intracellular life cycle and host innate responses.

After entering the host cell through endocytosis, Rickettsia bacteria undergo endolysosome escape and cytoplasmic replication, similarly to O. tsutsugamushi. However, these bacteria exit from the host cell through direct transfer to an adjacent cell (spotted fever group), focal lysis (spotted fever group), or cell lysis (typhus group). Host immune recognition is mediated by TLR2/4-regulated mechanisms, which activate the MyD88 pathway, or NLRP3/ASC, which activate the inflammasomes. Recognition of Rickettsia is followed by rapid transcription of proinflammatory cytokines and chemokines. 
R. akari, both TLR2- and TLR4-expressing HEK293T cells exhibited NF- $\mathrm{KB}$ activation, whereas TLR $2 / 4$ negative cells did not. Using a luciferase-based assay, the authors then showed that engineering specific amino acid residue mutations within TLR2 (R753Q) or TLR4 (D299G) abrogated NF- $\kappa B$ activation in HEK293T cells, thus further implicating the receptors in directly recognizing the bacterium. When anti-TLR2 and anti-TLR4 antibodies were used simultaneously in human monocytes exposed to heat-killed $R$. akari, the TNF $\alpha$ expression was found to be $\sim 40 \%$ that in control cells. However, the effects of these antibodies were modest in cells exposed to live R. akari. Live bacteria might potentially stimulate TNF $\alpha$ expression in a TLR 2/4dependent and -independent manner, and TLR 2 may play a minor role in immune responses to rickettsia. This speculation is supported by findings from a recent study revealing no differences in survival or bacterial loads between $R$. conorii infected TLR $2^{-/-}$and WT C57BL/6 mice [74].

Nevertheless, MyD88 is essential for host sensing and protection against Rickettsia. MyD88 ${ }^{-1-} \mathrm{C} 57 \mathrm{BL} / 6$ mice are highly susceptible to high-dose $R$. conorii ( $0 \%$ survival), as compared with WT mice (100\% survival), owing to uncontrolled bacterial growth in the liver, spleen, and lungs [74]. R. australis-infected MyD88 ${ }^{-/}$mice also display low expression of type 1 and proinflammatory cytokines in the lungs (IFN $\gamma$, TNF $\alpha$, IL-6, and IL-1 $\beta$ transcripts) and sera (IFN $\gamma$, IL-12-p40, IL-12-p70, IL-6, and G-CSF proteins). Histologic analysis has revealed decreased $M \Phi$ numbers and a diminished frequency/amount of inflammatory infiltration in the liver, concomitantly with fewer neutrophils in the lungs in infected $\mathrm{MyD} 88^{-/-}$animals. In vitro studies of infected MyD88 ${ }^{-1-}$ BMDCs, compared with WT cells, have revealed diminished MHC-II expression and no increase in IL-12-p40 expression, thus indicating impaired DC maturation. Together, these findings indicate that MyD88 is responsible for host protection against $R$. australis via DC maturation and the generation of type 1-skewed responses.

\section{NLR- and ASC-mediated immune recognition}

New evidence of how NLRs influence inflammasomes during rickettsial infection has recently emerged. Inflammasome activation can lead to different infection outcomes depending on the Rickettsia species. R. parkeriinduced inflammasome activation has been shown to antagonize type- 1 interferon responses in vitro and in vivo, thus allowing bacterial growth [79]. This phenomenon has been observed in infected Caspase 1/11 ${ }^{-/-}$C57BL/6 BMMФs, which exhibit lower bacterial loads and higher type 1 interferon than WT [79]. However, no specific NLR has been implicated in driving these findings. In contrast, inflammasomes inhibit $R$. australis growth. $R$. australis-infected human peripheral blood mononuclear cell (PBMC)-derived M $\mathrm{Ms}$ and $\mathrm{C} 57 \mathrm{BL} / 6$ BMMФs rapidly secrete IL- $1 \beta$ as early as 3 hpi [80]. Concomitantly, NLRP3 transcripts have been found to significantly increase in infected cells by 4 hpi, thus prompting further examination of the role of NLRP3 and ASC (a key NLR-inflammasome adaptor protein) in recognizing $R$. australis infection. NLRP3 ${ }^{-/-}$BMMФs exhibit decreased IL- $1 \beta$ secretion across different infectious doses, whereas cleaved Caspase-1 (indicating inflammasome activation) is evident only at high doses. In in vivo studies, infected NLRP3 ${ }^{-/-}$mice, compared with WT mice, have been found to bear high bacterial loads in the spleen, but not the liver or lungs. The lack of effects on survival or histopathology in NLRP3 ${ }^{-/}$mice indicates that although NLRP3 may contribute to tissue specific responses, it is not essential for controlling infection in vivo. $\mathrm{ASC}^{-/-} \mathrm{M} \Phi$ s infected with $R$. australis have been found to produce virtually no IL- $1 \beta$, IL-18, or activated Caspase-1 protein; however, follow-up in vivo characterization is lacking. A subsequent study analyzing the role of inflammasome activation during $R$. australis infection has revealed a powerful role of ASC and potential crosstalk with TLR4 [77]. ASC ${ }^{-/-}$C57BL/6 mice infected with a sublethal dose of $R$. australis are highly susceptible to infection: $90 \%$ of these animals succumb to disease. Bacterial loads in the liver, lung, and spleen are significantly greater in $\mathrm{ASC}^{-/-}$animals, thus indicating the role of ASC in host resistance against $R$. australis infection. Additionally, serum levels of IL-1 $\beta$, IL-18, and IFN $\gamma$ have been found to be significantly lower in infected $\mathrm{ASC}^{-/-}$mice than controls in the terminal phase of disease. Interestingly, infected $\mathrm{ASC}^{-/-}$ BMM $\Phi$ s bear greater bacterial loads and produce more pro-IL-1 $\beta$ than WT. Purified $R$. australis LPS has also been found to stimulate pro-IL-1 $\beta$ transcription in $\mathrm{ASC}^{-/-} \mathrm{M} \Phi$ s. Given that pro-IL-1 $\beta$ is not induced after treatment with $R$. australis LPS in TLR $4^{-/}$M $\Phi$ s, ASC-driven inflammation has been suggested to be triggered by TLR 4-mediated IL-1 $\beta$ production.

\section{Knowledge gaps and future studies}

Although TLR4 is likely to be a key innate sensor for Rickettsia (Fig 2), defining its molecular interactions is not straightforward for several reasons. Rickettsia spp. have very low levels of LPS (1-2\% of total biomass) [81]. Given the inherent challenges in cultivating this obligate intracellular bacterium at a large scale, extraction and purification of rickettsial LPS in sufficient quantities for in vitro and in vivo analysis remains exceedingly difficult. Consequently, very few comparative studies have analyzed structural or immunologic differences between rickettsial LPS and the LPS from other Gram-negative bacteria, such as E. coli or Salmonella $[81,82]$. Therefore, bioinformatic and modeling studies are needed to predict structural interactions between rickettsial ligands and TLRs. Despite the involvement of NLRP3 and ASC during rickettsial infection, upstream mediators of inflammasomes, including ROS production and ion imbalance, have not been explored [80]. Whether inflammasome activation through NLRP3 and ASC occurs via host DAMP molecules, bacterial components/pathways, or other indirect signals also remains to be addressed. More studies examining innate recognition of Rickettsia are greatly needed, because most research has focused on relatively few species in the context of several sensing receptors. Furthermore, studies examining other classes of PRRs, 
including the CLRs and RLRs, in recognizing rickettsiae are lacking. Whether modes of innate recognition are universal across SFG, transitional group, and typhus group rickettsia remains to be determined.

\section{ANAPLASMA PHAGOCYTOPHILUM}

\section{Epidemiology and clinical features}

A. phagocytophilum is the etiologic agent of human granulocytic anaplasmosis, a potentially lethal febrile illness endemic to the northeastern and northern central United States [35]. Anaplasma spp. were once considered Ehrlichia and are closely associated with the genus Rickettsia $[36,83,84]$. Epidemiological surveys have revealed that Anaplasma spp. are maintained in a large pool of hosts, ranging from small mammals and birds to large mammals, including various species of deer and even horses [85]. Transmission occurs through the bites of Ixodes ticks, and humans are the accidental dead-end host [86]. Co-infection is common, because Ixodes ticks may also transmit Babesia microti, Borrelia burgdorferii, and encephalitic viruses [87]. Anaplasma genetic material has also been detected in sequenced saliva from Amblyomma and Dermacentor ticks; however, transmission from these vectors has not been extensively studied [88]. After infection, early clinical symptoms are nonspecific and include fever, chills, headache, and myalgias. In a small percentage of cases that are not properly treated $(<1 \%)$, HGA can lead to hematological issues, along with outcomes including respiratory distress, renal failure, and septic shock [89]. Although the clinical course of this disease has been characterized, the host immune recognition remains less clear. Given that the incidence rate of human granulocytic anaplasmosis in the United States has increased since 2008 [90,91], careful examination of the host immunological response is warranted.

\section{Immune recognition}

A. phagocytophilum is a Gram-negative bacterium that preferentially infects neutrophils [92]. After neutrophil invasion, intracytoplasmic replication occurs in clusters of bacteria known as morulae, which can be identified through blood smear approaches [93]. The many cellular events initiated by the bacteria prevent its detection and elimination. Previous reports have shown that inhibition of apoptosis occurs via the stimulation of the PI3K/Akt and p38 MAPK pathways, thus prolonging the survival of infected cells $[94,95]$. A. phagocytophilum also lacks genes necessary for LPS and peptidoglycan synthesis, thus facilitating passive immune evasion [83].

Early reports have suggested a possible role of TLR2 and MyD88 in sensing A. phagocytophilum in vitro [96,97]. Recently, the link between MyD88- or TRIF-dependent TLRs and inflammation has been explored via in vitro and in vivo approaches. Infected $\mathrm{MyD}^{-/-} 8^{-1} \mathrm{MyD} 88 /$ $\mathrm{TRIF}^{-/-}$, and TLR2/3/4/7/9 ${ }^{-/-}$murine Hoxb8 neutrophils, compared with WT cells, show diminished proinflammatory responses (Nos2 transcripts; TNFo, CCL4, and
CCL5 secretion) [98]. Despite differences in inflammation in vitro, no phenotypic differences have been observed in vivo between infected WT C57BL/6 and $\mathrm{TRIF}^{-/-}$mice. Collectively, these studies imply that although both MyD88and TRIF-dependent TLRs may sense A. phagocytophilum, they do not influence the outcome of infection.

Several studies have suggested that NLR activation occurs in response to A. phagocytophilum. First, human primary neutrophils upregulate RIP2 transcripts within 4 hpi [99]. Infected RIP2 ${ }^{-1-}$ C57BL/6 mice, compared with WT, exhibit higher bacterial loads in the blood and delayed clearance of infection [99] and additionally display two-fold lower IFN $\gamma$ levels. Thus, NLRs signaling through RIP2 have been speculated to contribute to mounting of the Th1 response to Anaplasma [99]. A separate study by Müller and colleagues has built upon this hypothesis by analyzing the contributions of specific NLRs during infection [98]. The authors observed significantly increased $A$. phagocytophilum loads in the blood and lungs of NOD2 ${ }^{-/-} \mathrm{C} 57 \mathrm{BL} / 6$ mice throughout the course of infection, but both NOD2 $2^{-/-}$ and WT mice eventually cleared the bacterium at similar rates [98]. In experiments using Hoxb8 murine neutrophils, no differences in bacterial load or proinflammatory markers have been observed among infected WT, NOD1 $1^{-1-}$, $\mathrm{NOD}^{-/-}$, and NLRP3 ${ }^{-/-}$cells [98]. However, A. phagocytophilum has been shown to activate NLRC4 via a unique mechanism. A. phagocytophilum-infected ВММФs produce increased amounts of prostaglandins (PGE2, PGD2, and TXA2) and show increased activity of cyclooxygenase and phospholipase enzymes [100]. A unique feature of this pathway occurs downstream, in that increased levels of PGE2 lead to the initiation and activation of the NLRC4 inflammasome complex [100]. The known activators of this inflammasome pathway are flagellin and T3SS, both of which are absent in Anaplasma. How Anaplasma infection activates the NLRC4 inflammasome remains unclear.

The contributions of other PRRs in sensing A. phagocytophilum are less understood. No studies have examined RLRs, and a single study has revealed no role of CLRs. To examine the contributions of CLRs, one study has infected DAP12 ${ }^{-/-}, \mathrm{FcR} \gamma^{-/-}, \mathrm{SYK}^{\mathrm{del} / \mathrm{del}}$ C57BL6 mice with $A$. phagocytophilum and followed them for the duration of the disease [98]. No differences in survival or bacterial loads in the blood, spleen, or lung were observed among the WT and $\mathrm{DAP} 12^{-/-}, \mathrm{FcR} \gamma^{-/-}$, or $\mathrm{SYK}^{\mathrm{del} / \mathrm{del}}$ mice. Additionally, infected DAP12 $2^{-/-}$and FcR $\gamma^{-/-}$Hoxb8 neutrophils, compared with WT counterparts, exhibited no differences in bacterial loads or proinflammatory markers. Notably, Syk ${ }^{\mathrm{del} / \mathrm{del}}$ Hoxb8 neutrophils were not studied, because these cells could not be cultivated in sufficient quantities. Thus, CLRs do not significantly contribute to controlling A. phagocytophilum infection in vivo or generating neutrophil inflammation in vitro.

\section{Knowledge gaps and future studies}

Despite advances in the understanding of innate responses to $A$. phagocytophilum, many challenges remain. One major 
hurdle in defining immune signatures associated with severe disease is that lethal models of anaplasmosis have not yet been developed. Although murine models of disease, including $\mathrm{C} 57 \mathrm{BL} / 6$ and $\mathrm{BALB} / \mathrm{c}$, accurately mimic the pathologic features associated with human disease, infection is generally cleared within 20 days [101]. Even infections of immunodeficient models, including $\mathrm{SCID}^{-/-}$mice, are non-lethal [102]. Thus, immunologic differences between mild and severe infection may be unclear. Although TLRs were the first PRR family studied, they have been found to play a small role in vivo and in vitro $[98,103,104]$. Because Anaplasma spp. lack both peptidoglycan and LPS, the limited role of TLRs is unsurprising. Additionally, the strongest evidence of NLR involvement largely relies on findings from knockout of the adaptor protein RIP2 [99]. Specific NLRs contributing to the innate response remain undefined, and future studies are needed to identify subclasses of NLRs that may recognize A. phagocytophilum. Finally, studies of host innate responses in the absence of Ixodes ticks is a concern, because tick saliva can modulate or dampen initial immune responses to $A$. phagocytophilum infection. Although one study using BMMФs treated with saliva from Ixodes scapularis has reported decreased TLR and NLR signaling [87], this aspect of infection has not yet been widely addressed.

\section{EHRLICHIA CHAFFEENSIS}

\section{Epidemiology and clinical features}

E. chaffeensis is the causative agent of human monocytic ehrlichiosis, an emerging tick-borne illness found predominantly in the southeastern and southern central United States [36]. E. chaffeensis is maintained in white-tailed deer [105] and transmitted to humans via bites of the Lonestar tick (Amblyomma americanum) [106]. Early symptoms of the disease are nonspecific (fever, headache, myalgia, anorexia, and chills); however, approximately $40 \%$ of identified cases require hospitalization because of multiorgan failure, thus resulting in case fatality rates of 2-3\% [107]. Clinical findings during $E$. chaffeensis infection include leukopenia, thrombocytopenia, anemia, and elevated liver aminotransferases, which are often confused with symptoms of $A$. phagocytophilum infection, thus resulting in under-reporting and misdiagnosis [107].

\section{Immune recognition}

E. chaffeensis shares many microbiological features with A. phagocytophilum and other Rickettsiales. It is a Gramnegative, small coccus with primary tropism for monocytes and macrophages, but it may also infect hepatocytes and endothelial cells [108]. After entering the host cell via endocytosis, the bacterium differentiates from the infectious (dense core) form to the replicative (reticulate) form (Fig 3) $[36,107]$. E. chaffeensis is well adapted to subvert immune detection in both the host and vector, because it lacks genes for LPS or peptidoglycan biosynthesis, similarly to O. tsutsugamushi and A. phagocytophilum [109]. Thus, understanding

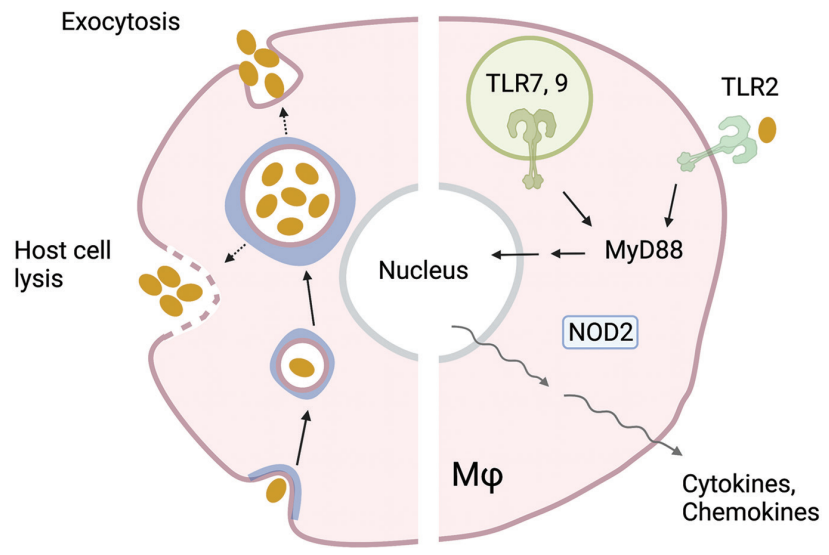

FIGURE 3 | Ehrlichia chaffeensis intracellular life cycle and host innate response.

Ehrlichia enters the cell via caveolae (blue)-mediated endocytosis. Initial entry is characterized by an infectious dense core, which later differentiates into a replicative reticulate form. Bacteria can exit from the cell through host cell lysis or exocytosis. Host immune recognition is mediated by TLR2 on the cell surface, or TLR7/TLR9 within the endosome, thereby leading to MyD88 pathway activation. NOD2 may also sense Ehrlichia within the cytoplasm. The phylogenetically related organism $A$. phagocytophilum induces a PRR activation signature similar to that of Ehrlichia, for innate recognition by TLR2 and NOD2. Together, TLR and NOD2 recognition activates the transcription of proinflammatory cytokines and chemokines.

of the immune recognition of this important pathogen has remained elusive.

TLRs are the most studied class of PRRs in the context of ehrlichial disease. One unique aspect observed from in vitro studies is that $E$. chaffeensis modulates TLR expression, thus enabling survival in host cells. For example, E. chaffeensis actively downregulates TLR2/TLR4 expression in human monocytes and monocyte-like THP-1 cells, partially through the action of $E$. chaffeensis secreted tandem repeat effector protein 120 [110]. In addition, the direct interaction of tandem repeat effector protein 120 with members of the Notch signaling pathway can lead to inhibition of PU.1,p38 MAPK, and ERK1/2, as well as increased bacterial survival [110]. Although E. chaffeensis modulates TLR2/TLR4 for its replication, the effect of this process on cytokine and chemokine signaling remains controversial. Miura et al. have shown that after infection with $E$. chaffeensis, BMMФs from TLR $2^{-/-}$or TLR $4^{-/-}$C57BL/6 mice produce significantly higher levels of CXCL2 transcripts (but not IL-1 $\beta$ and TNF $\alpha$ ) than WT controls [111]. Unexpectedly, MyD88 ${ }^{-/-}$ or MyD88/TRIF ${ }^{-/-}$MФs, HEK293 cells (lacking all known TLRs), or specific inhibitors of TLR3-, TLR7-, and TLR9mediated activation in the presence of E. chaffeensis infection have shown no major effects on cytokine/chemokine levels in comparison to controls [111]. Collectively, these in vitro studies suggest a limited role of TLRs in the expression of proinflammatory cytokines during infection.

The contribution of TLRs and NLRs in ehrlichial disease severity and pathogenesis have been examined by several groups [112-114]. A protective role of the MyD88mediated pathway in a murine model of mild ehrlichial 
disease was first reported by Koh et al. [112]: MyD88 $8^{-/-}$ mice bear greater bacterial loads in the blood and spleen, and have significantly lower serum IL-12 levels. Additionally, fewer apoptotic cells, lymphoblasts, $M \Phi_{\mathrm{s}}$, and neutrophils are observed in the spleen in infected $\mathrm{MyD} 88^{-/-}$animals. At the cellular level, E. muris infected MyD88 ${ }^{-/-}$BMDCs produce significantly fewer proinflammatory cytokines (IL-12-p40, TNF $\alpha$, and IL-6) than WT comparators. Unexpectedly, no differences in cytokine production have been observed among infected TLR2 $2^{-/-}, \mathrm{TLR}^{-/-}, \mathrm{TLR}^{-/-}, \mathrm{TLR}^{-/-}$, TLR $7^{-/}$, TLR $^{-/-}$, and TLR $11^{-/-}$DCs. Moreover, infection of $\mathrm{RIP}^{-/-}, \mathrm{NLRP}^{-/-}$, and $\mathrm{NLRC}^{-/-}$BMDCs has not revealed significant differences in bacterial loads or cytokine secretion. Therefore, each tested TLR and NLR by itself is insufficient for generating inflammatory cytokines in mild disease caused by E. muris infection.

However, the model of mild ehrlichial disease may not represent the immune signatures of severe infection. One study has addressed this aspect by comparing the roles of TLR 2 and NOD2 in both mild and severe forms of ehrlichial disease. Using infection with Ixodes ovatus Ehrlichia (IOE) to instigate lethal disease and E. muris as a model for mild disease, Chattoraj et al. have discovered the contributions of TLR2 and NOD2 in ameliorating or worsening pathogenesis [113]. At 3 dpi, transcripts of TLR2, TLR3, TLR 4, and TLR9 were all lower in the liver in both models than in mock controls, whereas the IOE-infected group had higher levels of NOD1 transcripts. However, by the terminal phase of IOE infection, TLR2 and MyD88 transcript levels were significantly higher than those in mock controls and E. muris counterparts. To evaluate the function of TLR 2 in lethal disease, the authors infected TLR $2^{-/-}$mice with IOE. TLR $2^{-1-}$ mice succumbed to disease more quickly than WT mice and showed greater hepatic bacterial loads, necrosis, and inflammatory foci at the end of the study [113]. In contrast, IOE-infected NOD2 $2^{-/-}$mice exhibited greater survival and hepatic bacterial clearance, along with fewer hepatic necrotic foci and apoptotic cells. These animals also displayed lower splenic $\mathrm{CD}^{+} \mathrm{T}$ cells, but higher natural killer $\mathrm{T}$ cells, CD4 $\mathrm{T}$ cells, Th1 signatures, and anti-inflammatory responses than did WT and TLR $2^{-/-}$mice [113]. Thus, TLR 2 may contribute to controlling infection, whereas NOD2 may enhance IOE-associated immunopathology.

Although the contribution of TLR2 has been emphasized, immunopathologic analysis of IOE-infected C57BL/6 mice has revealed a powerful role of TLR 9 in pathogenesis, inflammasome activation, and autophagy [114]. First, IOE-infected $\mathrm{MyD} 88^{-/-}$mice display greater survival and serum IL-10 levels than WT animals, despite their greater hepatic bacterial loads. MyD88 ${ }^{-/-}$mice also exhibit dampened hepatic injury and inflammasome activation. In a study examining the effect of TLR signaling on inflammasome activation at the cellular level, infected MyD88 ${ }^{-/-}$ ВMMФs have been found to secrete significantly fewer proinflammatory markers (IL-1 $\beta$, IL-1 $\alpha$, and TNF) than WT cells, whereas caspase- 1 cleavage and lactate dehydrogenase release are impaired. Markers indicating autophagy induction (Beclin-1 and Atg5) are also elevated in infected MyD88 ${ }^{-/-} M \Phi_{s}$, thus implicating TLRs in blunting autophagosome formation.TLR7 and TLR9 have been found to drive these findings, because IOE-infected TLR $7^{-/-} \mathrm{M} \Phi \mathrm{s}$ produce less IL-1 $\beta$ than WT, and infected TLR $9^{-/-} M \Phi$ s follow the same trend. However, Caspase- 1 and Caspase-11 activation are markedly hindered in infected TLR $9^{-/-}$cells, thereby implicating this receptor in inflammasome activation. Unexpectedly, IOE-infected TLR $9^{-/-}$mice have been found to be fully resistant to lethal ehrlichiosis (100\% survival) and to display less hepatic injury than WT controls [114]. The authors have demonstrated that accumulated mitochondrial DNA is the major TLR9 stimulus and have provided robust evidence that TLR9 is the key upstream mediator of MyD88-dependent effects [114].

\section{Knowledge gaps and future studies}

How do TLR2, TLR9, and NOD2 sense Ehrlichia during infection (Fig 3)? The rapid cytokine/chemokine responses to infection reported in multiple studies [111] suggest that bacterium-derived components are likely to activate these receptors directly. Proposed ligands forTLR2 include endogenous DAMPs or ehrlichial lipopeptides, whereas NOD2 may be stimulated by a low-molecular weight peptidoglycan homolog [113]. However, direct evidence supporting these interactions remains lacking. Despite considerable progress in understanding the TLR responses to Ehrlichia in vitro and in vivo, no published reports have examined other PRRs, including CLRs and RIG-I. Defining whether or how these additional receptors contribute to generating the immune response is necessary and could provide valuable insight into disease pathogenesis.

\section{COXIELLA BURNETII}

\section{Epidemiology and clinical features}

C. burnetii is the causative agent of "Q fever," which was first identified in Australia in 1937 [40]. Although C. burnetii is distributed across all continents except Antarctica (like Rickettsia spp.), cases of Q fever are most heavily clustered in regions containing livestock and farm animal processing centers [115]. Although the reservoir for this bacterium is expansive, comprising various animals, livestock are the most common source implicated in transmission to humans [37]. Inhalation of aerosolized bacteria from livestock birthing or still birth fluid, vaginal mucus, feces, and other secretions is the most common form of spread [116-118]. Ingestion of unpasteurized milk and cheese containing this bacterium is another mode of transmission but poses lower risks of infection $[118,119]$. Arthropods may play a role in transmission, because C. burnetii have been identified in various tick species, including Ixodes, Dermacentor, Rhipicephalus, and Haemaphysalis [38-40]. However, the roles of ticks in human infection and pathogenesis are unknown. Most acute $\mathrm{Q}$ fever cases are asymptomatic, but patients may also present with constitutional signs and symptoms, thus making accurate diagnosis difficult [120]. Furthermore, although 
rare (1-5\% of cases), chronic complications after primary infection may occur, including interstitial fibrosis, hepatitis, encephalitis, endocarditis, and valvular pathology [121,122].

\section{Immune recognition}

C. burnetii is a Gram-negative, LPS-positive, pleomorphic bacterium with a spore-like form [123]. Although classically considered a strict intracellular pathogen, this categorization may be revisited, owing to the successful cultivation C. burnetii in cell-free conditions $[124,125]$. For in vivo infection, alveolar macrophages or monocytes are the primary target cells for replication after inhalation into the host [126]. C. burnetii then replicates within phagolysosomes, forming a Coxiella containing vacuole (Fig 4). In contrast to other intracellular bacteria, which either escape the endosome or thwart phagolysosome acidification, Coxiella grows best in acidified vacuoles [127]. The life cycle of C. burnetii takes two forms: a stable small cell variant, which is capable of penetrating host cells and surviving in the environment, and a large cell variant, which is metabolically active and replicates in host cells [128]. After 5 days, the Coxiella containing vacuole reaches a size that occupies most of the cell volume, and the large cell variant population begins to transition back into the small cell variant, which then can infect other cells [129]. Although Coxiella remains within cells, the release of pathogenic factors (such as AnkG, CaeA, $\mathrm{CaeB}$, and IcaA) into the cytoplasm promotes an anti-apoptotic environment that prolongs the lifespan of infected cells [130]. Additionally, C. burnetii strains are divided into two phase variants for laboratory study, on the basis of virulence and LPS structures. Phase I variants are virulent, synthesize

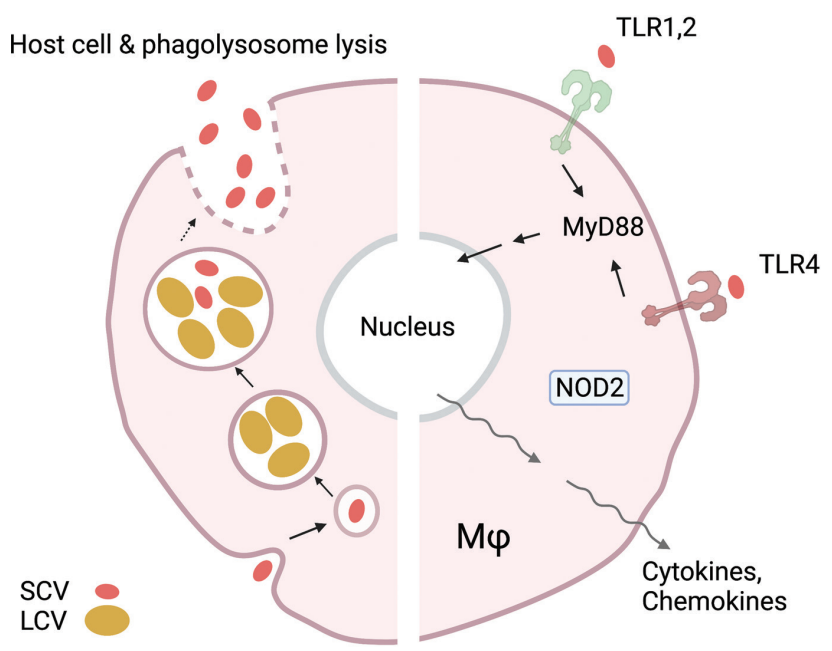

FIGURE 4 | Coxiella burnetii intracellular life cycle and host innate responses.

Coxiella exhibits two different morphologic variants during its life cycle. A stable small cell variant (SCV), which can penetrate the cell via endocytosis, and a large cell variant (LCV), which performs metabolic processes and replication. Only the SCV exits the cell via the host-cell-phagolysosome lysis. Host immune recognition is mediated by NOD2, as well as by TLR1/2/4-regulated activation of MyD88 or TRIF. Immune recognition of Coxiella then leads to the transcription of target proinflammatory cytokines and chemokines.
LPS containing highly branched O-chains, and generally are isolated from infected individuals or animals [131]. Phase II variants, in contrast, are avirulent, synthesize truncated O-antigen, and are generated via laboratory passage [132]. Phase II variants are immunostimulatory and capable of activating innate immune cells, whereas phase I variants are better able to evade innate recognition [133]. Differences in LPS structure may explain these findings: phase I variant LPS may mask other PAMPs on the surface of C. burnetii [133]. The ability of phase I variants to evade immune detection has been hypothesized to be linked to chronic Q fever infection [133], thus highlighting the importance of efforts to thoroughly understand the innate recognition of this bacterium.

\section{TLR/MyD88-mediated recognition}

Multiple studies have examined the roles of TLR1/2/4and MyD88-mediated pathways in recognizing C. burnetii, including reports on human participants or samples. Evidence from a case-control study has revealed a positive association between a single nucleotide polymorphism (SNP) in MyD88 (-938C >A) and the development of chronic Q fever [134]. However, people with the TLR1 R80T genotype are less likely to develop chronic disease. The functional consequence of these SNPs has been examined by stimulating whole blood with C. burnetii. Interestingly, infected whole blood from MyD88 (-938C > A) participants has been found to exhibit no differences in cytokine (IL$1 \beta$, TNF, IL-2, IL-6, and IL-10) production, whereas TLR1 R80T whole blood shows decreased IL-10 responses. Given that high serum IL-10 is a marker of poor prognosis in $\mathrm{Q}$ fever, this TLR1 mutation has functional relevance to chronic disease. Additional evidence of the contribution of TLRs has been provided by Ammerdorffer and colleagues, who have investigated the roles of TLR polymorphisms in human PBMCs [135]. First, PBMCs infected with C. burnetii Nine Mile (NM) or 3262 strains (phase I variants) were found to secrete high levels of proinflammatory cytokines (IL-1 $\beta$, TNF $\alpha$, and IL-6). For both strains, blocking TLR4 before infection did not produce reductions in any cytokine measured, whereas blocking TLR2 abrogated IL-1 $\beta$ and IL-6 secretion. PBMCs were then divided into groups on the basis of the presence of TLR SNPs and infected with C. burnetii NM and 3262. Polymorphisms of TLR4 were not associated with decreased cytokine production after infection, whereas PBMCs homozygous for TLR2 P631H displayed decreased IL-1 $\beta$ responses after infection with $C$. burnetii 3262 only [135]. Furthermore, PBMCs containing homozygous TLR1 variants showed significantly decreased production of both IL-1 $\beta$ and TNF $\alpha$ after infection with $C$. burnetii NM and 3262. A similar trend has been observed for PBMCs homozygous for the TLR6 P249S SNP, but only in the context of C. burnetii 3262. These findings were evaluated by using C57BL/6 BMM $\Phi$ s. Infected TLR $2^{-/-}$and $\mathrm{TLR}^{-/-}$cells were found to produce less IL-6 in response to both strains of C. burnetii tested, whereas TLR $1^{-/-}$cells produced less IL-6 only in response to C. burnetii NM. 
Collectively, these results reveal virulent $C$. burnetii strain specific differences in immune sensing, and shared contributions between TLR1/2 in human PBMCs.

Studies using animal models of $\mathrm{Q}$ fever have shed light on the roles of TLRs in the pathogenesis of infection. Multiple reports have shown that TLR2/4 and MyD88-mediated signaling is essential in controlling the replication of $C$. burnetii Nine Mile Phase II (NMII) infection in vitro and in vivo [136-138]. An early report using $\mathrm{CHO}$ cells transfected with functional TLR2 or TLR 4 has revealed that $C$. burnetii NMII activates only TLR $2^{+}$cells [138]. Moreover, $\mathrm{TLR}^{-/-}$C57BL/6 BMM ${ }^{-1}$ s have been found to display a significantly greater NMII load at 8 dpi than WT cells, along with virtually abolished TNF $\alpha$ and IL-12 secretion, even after treatment with doses as high as 500 bacteria per cell. Notably, TLR4-impaired (C3H/HeJ) MФs have not been found to exhibit any significant differences with respect to control $(\mathrm{C} 3 \mathrm{H} / \mathrm{HePas})$ cells. Because C. burnetii contains LPS, they the authors then asked why TLR 4 activation did not occur. To answer this question, the authors treated human PBMCs with purified LPS from phase I and phase II C. burnetii before the addition of E. coli endotoxin. From these experiments, the authors determined that PBMCs treated with C. burnetii phase I and phase II LPS exhibit blunted cytokine profiles in response to E. coli endotoxin, thus indicating that C. burnetii LPS may act antagonistically toward TLR4 [138]. These results suggest that TLR2, but notTLR4, is necessary for the regulation and modulation of pro-inflammatory responses in C. burnetii NMII infections.

A subsequent study by Bradley et al. has revealed that infected MyD88 ${ }^{-/-}$or TRIF ${ }^{-/-}$C57BL/6 BMMФs secrete significantly lower yet substantial levels of TNF $\alpha$ and IL-6 than WT cells [137]. In contrast, TNF $\alpha$ and IL-6 secretion is virtually abolished in infected MyD88/TRIF ${ }^{-/-} \mathrm{M} \Phi$ s, thus indicating that TLR responses to NMII rely on both signaling adaptors. Similarly, MyD88/TRIF ${ }^{-/}$cells, compared with other groups, are most permissive to infection, as evidenced by a greater number of intracellular vacuoles. Therefore, the authors examined the effects of TLR2 and TLR4, and observed that infected TLR2 $2^{-/-}$BMMФs secreted significantly reduced levels of TNF $\alpha$ and IL-6 after infection and bore increased bacterial loads, whereas $\mathrm{TLR}^{-/-}$cells showed no significant changes. However, infected TLR2/TLR $4^{-/-}$cells did not secrete detectable amounts of TNF $\alpha$ and IL-6, thus suggesting that both receptors engage in crosstalk and subsequently stimulate inflammation in response to NMII. Notably,TLR2/TLR $4^{-/-}$cells did not exhibit greater bacterial loads than WT controls. Together, these results imply a unique role of TLR2 in controlling bacterial infection and show the importance of TLR2-TLR 4 crosstalk in generating inflammatory profiles [137]. A separate study has revealed similar findings, showing that $\mathrm{MyD} 88^{-/-} \mathrm{M} \Phi$ s bear significantly higher bacterial loads at 72 hpi than WT controls, as well as diminished production of IL-6 and IL-10. Together, these results indicate a role of MyD88 in producing both a cytokine response and regulating the bacterial load in $M \Phi$ s [136]. In that study, after intratracheal infection, the bacterial load of C. burnetii
NMII was consistently higher in the lung, spleen, heart, and liver tissues from 7 to $120 \mathrm{dpi}$; in contrast, $\mathrm{MyD} 88^{+/-}$mice showed complete clearance at 27 dpi [136]. Despite harboring a greater bacterial burden, the MyD88 ${ }^{-/-}$mice did not show signs of disease or weight loss. Chemokine/cytokine expression and histopathologic analysis indicated that infected MyD88 ${ }^{-/-}$mice displayed reduced splenic CCL2 and IFN $\gamma$ responses that correlated with smaller granulomatous foci in the liver. Therefore, MyD88 is fundamental to the control of Coxiella NMII infection.

\section{NLR-mediated immune recognition}

Few studies have examined the contribution of NLRs during C. burnetii infection. Although one SNP in NOD2 (L1007fsX1) has been associated with the development of chronic Q fever, infection of human PBMCs has revealed no functional consequence of this variant [134]. However, a study analyzing multiple human NOD2 polymorphisms has revealed a functional effect on cytokine secretion in response to infection with two different phase I strains [135]. Human PBMCs homozygous for NOD2 3020insC secrete significantly less IL-1 $\beta$ and IL-6 in response to $C$. burnetii 3262, whereas nine other NOD2 SNPs have been found to have no effects on inflammatory responses [135]. Infected PBMCs bearing NOD1 polymorphisms have also been found to exhibit cytokine/chemokine profiles similar to those of control PBMCs. In C57BL/6 MФs, C. burnetii $\mathrm{NM}$ stimulation of $\mathrm{NOD}^{-/-}$cells has been found to result in a $35 \%$ decrease in IL-6 secretion and 50\% decrease in $\mathrm{NOD}^{-/-}$cells [135]. Together, these studies suggest a role of NOD2 in sensing C. burnetii.

\section{Knowledge gaps and future studies}

The consensus on the basis of available data reveals a powerful role of TLR2 in sensing C. burnetii (Fig 4). Studies using human samples and animal models of infection consistently indicate that TLR2 contributes to generating inflammation and controlling bacterial replication [134-138]. The cytokine response has also been shown to involve crosstalk between TLR 2 and TLR4, wherein the effect of TLR4 depends on TLR 2 [137].TLR1 may also play a role in infection, and TLR2 homodimers and TLR1/TLR2 heterodimers can recognize Coxiella [135]. Although no study to date has revealed the natural ligands involved, components of this bacterium's lipoprotein-rich cell wall are the most likely culprit. The observation that C. burnetii LPS may be immunosuppressive similarly to Bartonella is also consistent with findings from studies revealing no role of TLR 4 alone in contributing to inflammation and controlling infection [138]. Although NOD2 is likely to be involved in sensing this bacterium after it has been engulfed, studies examining pathogenesis in NOD2 $2^{-1-}$ mice have yet to be performed. Furthermore, evaluation and identification of the natural ligand for NOD2 are needed. Mechanistic studies to identify such ligands may be aided by newly established axenic culturing techniques for C. burnetii, which allow for largescale bacterial propagation $[124,125]$. Finally, the effects of RLRs and CLRs have not been evaluated for C. burnetii. 
Careful assessment of the potential roles of both receptor families may yield valuable insight into innate recognition.

\section{CONCLUDING REMARKS}

Rickettsia, O. tsutsugamushi, Anaplasma, Ehrlichia, and C. burnetii cause substantial human disease worldwide, yet understanding of the mechanisms of innate recognition remains a challenge. Despite sharing aspects of basic biology, each of these five bacteria exhibit unique characteristics, tropisms, and natural reservoirs (Table 1), which may influence immune recognition. To date, research efforts have emphasized better-known PRRs, particularly TLRs and NLRs, while neglecting RLRs or CLRs, possibly because TLR- and NLR-deficient mouse strains and reagents are widely available, whereas those for CLRs are severely lacking. However, studies examining the effects of CLRs may provide insight. Given that CLRs sense both PAMPs and DAMPs, defining the roles of these receptors in the recognition of obligate intracellular bacteria, as in the case of $O$. tsutsugamushi with Mincle, may lead to new fields of investigation. Continued research on the recognition of obligate intracellular bacteria would improve understanding of disease pathogenesis and might lead to new therapeutic strategies for patients with severe disease. Additionally, because the United States does not have licensed vaccines for any of the five bacteria discussed herein, evaluating how PRRs influence the adaptive response to infection could provide valuable information for future vaccine design.

\section{ACKNOWLEDGEMENTS}

All figures were generated with the BioRender platform. This work was partially supported by National Institute of Allergy and Infectious Diseases grants (R01 Al132674 to LS, T32 Al007526-20 to LS, and R21 Al156536-01 to LS, https://www.niaid.nih.gov), a UTMB Center for Biodefense and Emerging Infectious Diseases Pilot grant (to LS, https:// www.utmb.edu/cbeid), and Department of Defense Threat Reduction Agency grant HDTRA1-19-1-0043 (subaward to LS, https://www.dtra. mil/). JF was a recipient of a NIAID Emerging and Tropical Infectious Diseases T32 fellowship. ZC was a recipient of a NIAID Infectious Diseases and Inflammatory Disorders Training Program T35 fellowship (Al078878). The funders had no role in the study design, data collection and analysis, decision to publish, or preparation of the manuscript.

\section{COMPETING INTERESTS}

Professor Lynn Soong is co-Editor-in-Chief of Zoonoses. She was not involved in the peer-review or handling of the manuscript. The authors have no other competing interests to disclose.

\section{REFERENCES}

1. Akira S, Takeda K. Toll-like receptor signalling. Nat Rev Immunol. 2004;4(7):499-511.

2. Fitzgerald KA, Kagan JC. Toll-like receptors and the control of immunity. Cell. 2020;180(6):1044-1066.

3. Elinav E, Strowig T, Henao-Mejia J, Flavell RA. Regulation of the antimicrobial response by NLR proteins. Immunity. 2011;34(5):665-679.

4. Rehwinkel J, Gack MU. RIG-I-like receptors: their regulation and roles in RNA sensing. Nat Rev Immunol. 2020;20(9):537-551.
5. Brown GD, Willment JA, Whitehead L. C-type lectins in immunity and homeostasis. Nat Rev Immunol. 2018;18(6):374-389.

6. Medzhitov R, Preston-Hurlburt P, Janeway CA, Jr. A human homologue of the Drosophila Toll protein signals activation of adaptive immunity. Nature. 1997;388(6640):394-397.

7. Kang JY, Nan X, Jin MS, Youn SJ, Ryu YH, Mah S, et al. Recognition of lipopeptide patterns by Toll-like receptor 2-Toll-like receptor 6 heterodimer. Immunity. 2009;31(6):873-884.

8. Takeuchi O, Kawai T, Sanjo H, Copeland NG, Gilbert DJ, Jenkins NA, et al. TLR6: a novel member of an expanding toll-like receptor family. Gene. 1999;231(1-2):59-65.

9. Campos MA, Almeida IC, Takeuchi O, Akira S, Valente EP, Procopio DO, et al. Activation of Toll-like receptor-2 by glycosylphosphatidylinositol anchors from a protozoan parasite. J Immunol. 2001;167(1):416-423.

10. Takeuchi O, Sato S, Horiuchi T, Hoshino K, Takeda K, Dong Z, et al. Cutting edge: role of Toll-like receptor 1 in mediating immune response to microbial lipoproteins. J Immunol. 2002;169(1):10-14.

11. Poltorak A, Smirnova I, He X, Liu MY, van Huffel C, McNally $O$, et al. Genetic and physical mapping of the Lps locus: identification of the toll-4 receptor as a candidate gene in the critical region. Blood Cells Mol Dis. 1998;24(3):340-355.

12. Gewirtz AT, Navas TA, Lyons S, Godowski PJ, Madara JL. Cutting edge: bacterial flagellin activates basolaterally expressed TLR5 to induce epithelial proinflammatory gene expression. J Immunol. 2001;167(4):1882-1885.

13. Alexopoulou L, Holt AC, Medzhitov R, Flavell RA. Recognition of double-stranded RNA and activation of NF-kappaB by Toll-like receptor 3. Nature. 2001;413(6857):732-738.

14. Diebold SS, Kaisho T, Hemmi H, Akira S, Reis e Sousa C. Innate antiviral responses by means of TLR7-mediated recognition of single-stranded RNA. Science. 2004;303(5663):1529-1531.

15. Greulich W, Wagner M, Gaidt MM, Stafford C, Cheng Y, Linder A, et al. TLR8 is a sensor of RNase T2 degradation products. Cell. 2019;179(6):1264-1275 e13.

16. Heil F, Hemmi H, Hochrein $H$, Ampenberger F, Kirschning C, Akira $S$, et al. Species-specific recognition of singlestranded RNA via toll-like receptor 7 and 8 . Science. 2004;303(5663):1526-1529.

17. Ito T, Amakawa R, Kaisho T, Hemmi H, Tajima K, Uehira K, et al. Interferon-alpha and interleukin-12 are induced differentially by Toll-like receptor 7 ligands in human blood dendritic cell subsets. J Exp Med. 2002;195(11):1507-1512.

18. Hemmi H, Takeuchi O, Kawai T, Kaisho T, Sato S, Sanjo H, et al. A toll-like receptor recognizes bacterial DNA. Nature. 2000;408(6813):740-745.

19. Mohammad Hosseini A, Majidi J, Baradaran B, Yousefi M. Tolllike receptors in the pathogenesis of autoimmune diseases. Adv Pharm Bull. 2015;5(Suppl 1):605-614.

20. Farrugia M, Baron B. The role of toll-like receptors in autoimmune diseases through failure of the self-recognition mechanism. Int J Inflam. 2017;2017:8391230.

21. Fritz JH, Ferrero RL, Philpott DJ, Girardin SE. Nod-like proteins in immunity, inflammation and disease. Nat Immunol. 2006;7(12):1250-1257.

22. Coulombe F, Divangahi M, Veyrier F, de Leseleuc L, Gleason JL, Yang Y, et al. Increased NOD2-mediated recognition of N-glycolyl muramyl dipeptide. J Exp Med. 2009;206(8):1709-1716.

23. Franchi L, Amer A, Body-Malapel M, Kanneganti TD, Ozoren N, Jagirdar $\mathrm{R}$, et al. Cytosolic flagellin requires Ipaf for activation of caspase-1 and interleukin 1beta in salmonella-infected macrophages. Nat Immunol. 2006;7(6):576-582.

24. Franchi L, Stoolman J, Kanneganti TD, Verma A, Ramphal R, Nunez G. Critical role for Ipaf in Pseudomonas 
aeruginosa-induced caspase-1 activation. Eur J Immunol. 2007;37(11):3030-3039.

25. Miao EA, Andersen-Nissen E, Warren SE, Aderem A. TLR5 and Ipaf: dual sensors of bacterial flagellin in the innate immune system. Semin Immunopathol. 2007;29(3):275-288.

26. Miao EA, Ernst RK, Dors M, Mao DP, Aderem A. Pseudomonas aeruginosa activates caspase 1 through Ipaf. Proc Natl Acad Sci U S A. 2008;105(7):2562-2567.

27. Goubau D, Deddouche S, Reis e Sousa C. Cytosolic sensing of viruses. Immunity. 2013;38(5):855-869.

28. Zhao Y, Ye X, Dunker W, Song Y, Karijolich J. RIG-I like receptor sensing of host RNAs facilitates the cell-intrinsic immune response to KSHV infection. Nat Commun. 2018;9(1):4841.

29. Chiang JJ, Sparrer KMJ, van Gent M, Lassig C, Huang T, Osterrieder $\mathrm{N}$, et al. Viral unmasking of cellular $5 \mathrm{~S}$ rRNA pseudogene transcripts induces RIG-I-mediated immunity. Nat Immunol. 2018;19(1):53-62.

30. Geijtenbeek TB, Gringhuis SI. Signalling through C-type lectin receptors: shaping immune responses. Nat Rev Immunol. 2009;9(7):465-479

31. Salje J. Cells within cells: rickettsiales and the obligate intracellular bacterial lifestyle. Nat Rev Microbiol. 2021;19(6):375-390.

32. Blanton LS. The rickettsioses: a practical update. Infect Dis Clin North Am. 2019;33(1):213-229.

33. Abdad MY, Abou Abdallah R, Fournier PE, Stenos J, Vasoo $S$. A concise review of the epidemiology and diagnostics of rickettsioses: Rickettsia and Orientia spp. J Clin Microbiol. 2018;56(8):e01728-e01817.

34. Xu G, Walker DH, Jupiter D, Melby PC, Arcari CM. A review of the global epidemiology of scrub typhus. PLoS Negl Trop Dis. 2017;11(11):e0006062.

35. Bakken JS, Dumler JS. Human granulocytic anaplasmosis. Infect Dis Clin North Am. 2015;29(2):341-355.

36. Paddock CD, Childs JE. Ehrlichia chaffeensis: a prototypical emerging pathogen. Clin Microbiol Rev. 2003;16(1):37-64.

37. Kalaitzakis E, Fancello T, Simons X, Chaligiannis I, Tomaiuolo $\mathrm{S}$, Andreopoulou $\mathrm{M}$, et al. Coxiella burnetii Shedding in milk and molecular typing of strains infecting dairy cows in greece. Pathogens. 2021;10(3):287.

38. Spitalska E, Sparagano O, Stanko M, Schwarzova K, Spitalsky Z, Skultety L, et al. Diversity of Coxiella-like and Francisellalike endosymbionts, and Rickettsia spp., Coxiella burnetii as pathogens in the tick populations of Slovakia, Central Europe. Ticks Tick Borne Dis. 2018;9(5):1207-1211.

39. Truong AT, Yun BR, Lim J, Min S, Yoo MS, Yoon SS, et al. Realtime PCR biochip for on-site detection of Coxiella burnetii in ticks. Parasit Vectors. 2021;14(1):239.

40. Eldin C, Melenotte C, Mediannikov O, Ghigo E, Million M, Edouard S, et al. From Q Fever to Coxiella burnetii Infection: a Paradigm Change. Clin Microbiol Rev. 2017;30(1):115-190.

41. Salje J. Orientia tsutsugamushi: a neglected but fascinating obligate intracellular bacterial pathogen. PLoS Pathog. 2017;13(12):e1006657.

42. Baker S, Dougan G. The genome of Salmonella enterica serovar Typhi. Clin Infect Dis. 2007;45(Suppl 1):S29-S33.

43. Blattner FR, Plunkett G, 3rd, Bloch CA, Perna NT, Burland V, Riley $\mathrm{M}$, et al. The complete genome sequence of Escherichia coli K-12. Science. 1997;277(5331):1453-1462.

44. Gorvel L, Textoris J, Banchereau R, Ben Amara A, Tantibhedhyangkul W, von Bargen $\mathrm{K}$, et al. Intracellular bacteria interfere with dendritic cell functions: role of the type I interferon pathway. PLoS One. 2014;9(6):e99420.

45. Samuel JE, Hendrix LR. Laboratory maintenance of Coxiella burnetii. Curr Protoc Microbiol. 2009; Chapter 6:Unit 6C 1.

46. Mansueto P, Vitale G, Cascio A, Seidita A, Pepe I, Carroccio $A$, et al. New insight into immunity and immunopathology of Rickettsial diseases. Clin Dev Immunol. 2012;2012:967852
47. Brouqui P, Dumler JS, Raoult D. Immunohistologic demonstration of Coxiella burnetii in the valves of patients with Q fever endocarditis. Am J Med. 1994;97(5):451-458.

48. Melenotte C, Protopopescu C, Million M, Edouard S, Carrieri MP, Eldin C, et al. Clinical features and complications of Coxiella burnetii infections from the French National Reference Center for Q Fever. JAMA Netw Open. 2018;1(4):e181580.

49. Fisher J, Card G, Liang Y, Trent B, Rosenzweig H, Soong L. Orientia tsutsugamushi selectively stimulates the C-type lectin receptor Mincle and type 1-skewed proinflammatory immune responses. PLoS Pathog. 2021;17(7):e1009782.

50. Soong L, Wang H, Shelite TR, Liang Y, Mendell NL, Sun J, et al. Strong type 1, but impaired type 2, immune responses contribute to Orientia tsutsugamushi-induced pathology in mice. PLoS Negl Trop Dis. 2014;8(9):e3191.

51. Weitzel T, Jiang J, Acosta-Jamett G, Martinez-Valdebenito C, Lopez J, Richards AL, et al. Canine seroprevalence to Orientia species in southern Chile: a cross-sectional survey on the Chiloe Island. PLoS One. 2018;13(7):e0200362.

52. Weitzel T, Acosta-Jamett G, Jiang J, Martinez-Valdebenito C, Farris CM, Richards AL, et al. Human seroepidemiology of Rickettsia and Orientia species in Chile -a cross-sectional study in five regions. Ticks Tick Borne Dis. 2020;11(6):101503.

53. Ghorbani RP, Ghorbani AJ, Jain MK, Walker DH. A case of scrub typhus probably acquired in Africa. Clin Infect Dis. 1997;25(6):1473-1474.

54. Osuga K, Kimura M, Goto H, Shimada K, Suto T. A case of Tsutsugamushi disease probably contracted in Africa. Eur J Clin Microbiol Infect Dis. 1991;10(2):95-96.

55. Walker JS, Gan E, Chan Teik C, Muul I. Involvement of small mammals in the transmission of scrub typhus in Malaysia: isolation and serological evidence. Trans R Soc Trop Med Hyg. 1973;67(6):838-845.

56. Phasomkusolsil $S$, Tanskul $P$, Ratanatham $S$, Watcharapichat $P$, Phulsuksombati D, Frances SP, et al. Transstadial and transovarial transmission of Orientia tsutsugamushi in Leptotrombidium imphalum and Leptotrombidium chiangraiensis (Acari: Trombiculidae). J Med Entomol. 2009;46(6):1442-1445.

57. Taylor AJ, Paris DH, Newton PN. A systematic review of mortality from untreated scrub typhus (Orientia tsutsugamushi). PLoS Negl Trop Dis. 2015;9(8):e0003971.

58. Bonell A, Lubell $Y$, Newton PN, Crump JA, Paris DH. Estimating the burden of scrub typhus: A systematic review. PLoS Negl Trop Dis. 2017;11(9):e0005838.

59. Dittrich S, Card E, Phuklia W, Rudgard WE, Silousok J, Phoumin P, et al. Survival and growth of Orientia tsutsugamushi in conventional hemocultures. Emerg Infect Dis. 2016;22(8):1460-1463.

60. Evans SM, Rodino KG, Adcox HE, Carlyon JA. Orientia tsutsugamushi uses two Ank effectors to modulate NF-אB p65 nuclear transport and inhibit NF- $\mathrm{KB}$ transcriptional activation. PLoS Pathog. 2018;14(5):e1007023.

61. Janardhanan J, Joseph Martin S, Astrup E, Veeramanikandan R, Aukrust P, Abraham OC, et al. Single-nucleotide polymorphisms in Toll-like receptor (TLR)-2, TLR4 and heat shock protein 70 genes and susceptibility to scrub typhus. J Hum Genet. 2013:58(11):707-710.

62. Najmi N, Kaur G, Sharma SK, Mehra NK. Human tolllike receptor 4 polymorphisms TLR4 Asp299Gly and Thr399lle influence susceptibility and severity of pulmonary tuberculosis in the Asian Indian population. Tissue Antigens. 2010;76(2):102-109.

63. Gharaibeh $M$, Hagedorn M, Lilla S, Hauptmann M, Heine $\mathrm{H}$, Fleischer $\mathrm{B}$, et al. Toll-like receptor 2 recognizes Orientia tsutsugamushi and increases susceptibility to murine experimental scrub typhus. Infect Immun. 2016;84(12):3379-3387.

64. Min CK, Kim HI, Ha NY, Kim Y, Kwon EK, Yen NTH, et al. A type I interferon and IL-10 induced by Orientia tsutsugamushi 
infection suppresses antigen-specific T cells and their memory responses. Front Immunol. 2018;9:2022.

65. Rodino KG, Adcox HE, Martin RK, Patel V, Conrad DH, Carlyon JA. The obligate intracellular bacterium Orientia tsutsugamushi targets NLRC5 to modulate the major histocompatibility complex class I pathway. Infect Immun. 2019;87(3):e00876-e00918.

66. Cho KA, Jun YH, Suh JW, Kang JS, Choi HJ, Woo SY. Orientia tsutsugamushi induced endothelial cell activation via the NOD1-IL-32 pathway. Microb Pathog. 2010;49(3):95-104

67. Koo JE, Hong HJ, Dearth A, Kobayashi KS, Koh YS. Intracellular invasion of Orientia tsutsugamushi activates inflammasome in asc-dependent manner. PLoS One. 2012;7(6):e39042.

68. Soong L. Dysregulated Th1 immune and vascular responses in scrub typhus pathogenesis. J Immunol. 2018;200(4):1233-1240.

69. Trent $B$, Liang $Y$, Xing $Y$, Esqueda $M$, Wei $Y, C$ ho NH, et al. Polarized lung inflammation and Tie2/angiopoietin-mediated endothelial dysfunction during severe Orientia tsutsugamushi infection. PLoS Negl Trop Dis. 2020;14(3):e0007675.

70. Astrup E, Janardhanan J, Otterdal K, Ueland T, Prakash JA, Lekva T, et al. Cytokine network in scrub typhus: high levels of interleukin- 8 are associated with disease severity and mortality. PLoS Negl Trop Dis. 2014;8(2):e2648.

71. Bora T, Khan SA. Evaluation of Th1 and Th2 immune response in clinical and sub-clinical scrub typhus infection. Hum Immunol. 2019;80(7):503-509.

72. Walker $\mathrm{DH}$, Ismail $\mathrm{N}$. Emerging and re-emerging rickettsioses: endothelial cell infection and early disease events. Nat Rev Microbiol. 2008;6(5):375-386.

73. Dumler JS, Walker DH. Rocky Mountain spotted fever changing ecology and persisting virulence. N Engl J Med. 2005;353(6):551-553.

74. Bechelli J, Smalley C, Zhao X, Judy B, Valdes P, Walker DH, et al. MyD88 mediates instructive signaling in dendritic cells and protective inflammatory response during rickettsial infection. Infect Immun. 2016;84(4):883-893.

75. Voss OH, Gillespie JJ, Lehman SS, Rennoll SA, Beier-Sexton M, Rahman MS, et al. Risk1, a phosphatidylinositol 3-kinase effector, promotes Rickettsia typhi intracellular survival. mBio. 2020;11(3):e00820-20.

76. Jordan JM, Woods ME, Soong L, Walker DH. Rickettsiae stimulate dendritic cells through toll-like receptor 4, leading to enhanced NK cell activation in vivo. J Infect Dis. 2009;199(2):236-242.

77. Rumfield C, Hyseni I, McBride JW, Walker DH, Fang R. Activation of ASC inflammasome driven by toll-like receptor 4 contributes to host immunity against rickettsial infection. Infect Immun. 2020;88(4):e00886-e00919.

78. Quevedo-Diaz MA, Song C, Xiong Y, Chen H, Wahl LM, Radulovic $S$, et al. Involvement of TLR2 and TLR4 in cell responses to Rickettsia akari. J Leukoc Biol. 2010;88(4):675-685.

79. Burke TP, Engstrom $P$, Chavez RA, Fonbuena JA, Vance RE, Welch MD. Inflammasome-mediated antagonism of type I interferon enhances Rickettsia pathogenesis. Nat Microbiol. 2020;5(5):688-696

80. Smalley C, Bechelli J, Rockx-Brouwer D, Saito T, Azar SR, Ismail N, et al. Rickettsia australis activates inflammasome in human and murine macrophages. PLoS One. 2016;11(6):e0157231

81. Fodorova M, Vadovic P, Skultety L, Slaba K, Toman R. Structural features of lipopolysaccharide from Rickettsia typhi: the causative agent of endemic typhus. Ann N Y Acad Sci. 2005; 1063:259-260

82. Fodorova M, Vadovic $P$, Toman R. Structural features of lipid $A$ of Rickettsia typhi. Acta Virol. 2011;55(1):31-44

83. Dumler JS, Asanovich KM, Bakken JS. Analysis of genetic identity of North American Anaplasma phagocytophilum strains by pulsed-field gel electrophoresis. J Clin Microbiol. 2003;41(7):3392-3394
84. Dumler JS, Barbet AF, Bekker CP, Dasch GA, Palmer GH, Ray SC, et al. Reorganization of genera in the families Rickettsiaceae and Anaplasmataceae in the order Rickettsiales: unification of some species of Ehrlichia with Anaplasma, Cowdria with Ehrlichia and Ehrlichia with Neorickettsia, descriptions of six new species combinations and designation of Ehrlichia equi and 'HGE agent' as subjective synonyms of Ehrlichia phagocytophila. Int I Syst Evol Microbiol. 2001;51(Pt 6):2145-2165.

85. Matei IA, D'Amico G, lonica AM, Kalmar Z, Corduneanu A, Sandor $A D$, et al. New records for Anaplasma phagocytophilum infection in small mammal species. Parasit Vectors. 2018;11(1):193.

86. Atif FA. Anaplasma marginale and Anaplasma phagocytophilum: rickettsiales pathogens of veterinary and public health significance. Parasitol Res. 2015;114(11):3941-3957.

87. Chen G, Severo MS, Sohail M, Sakhon OS, Wikel SK, Kotsyfakis $M$, et al. Ixodes scapularis saliva mitigates inflammatory cytokine secretion during Anaplasma phagocytophilum stimulation of immune cells. Parasit Vectors. 2012;5:229

88. Battilani M, De Arcangeli S, Balboni A, Dondi F. Genetic diversity and molecular epidemiology of Anaplasma. Infect Genet Evol. 2017;49:195-211.

89. Ismail N, Bloch KC, McBride JW. Human ehrlichiosis and anaplasmosis. Clin Lab Med. 2010;30(1):261-292.

90. Dahlgren FS, Heitman KN, Drexler NA, Massung RF, Behravesh CB. Human granulocytic anaplasmosis in the United States from 2008 to 2012: a summary of national surveillance data. Am J Trop Med Hyg. 2015;93(1):66-72.

91. Baker A, Wang HH, Mogg M, Derouen Z, Borski J, Grant WE. Increasing incidence of anaplasmosis in the United States, 2012 Through 2016. Vector Borne Zoonotic Dis. 2020;20(11):855-859.

92. Gussmann K, Kirschnek S, von Loewenich FD. Interferongamma-dependent control of Anaplasma phagocytophilum by murine neutrophil granulocytes. Parasit Vectors. 2017; 10(1):329.

93. Park HS, Shin KS, Son BR, Kim DM, Kim HS, Jeong HW. Human granulocytic anaplasmosis diagnosed based on a peripheral blood smear test in South Korea: a case report. Jpn J Infect Dis. 2020;73(6):469-472

94. Sarkar A, Hellberg L, Bhattacharyya A, Behnen M, Wang K, Lord JM, et al. Infection with Anaplasma phagocytophilum activates the phosphatidylinositol 3-Kinase/Akt and NF-KB survival pathways in neutrophil granulocytes. Infect Immun. 2012;80(4):1615-1623.

95. Choi KS, Park JT, Dumler JS. Anaplasma phagocytophilum delay of neutrophil apoptosis through the p38 mitogenactivated protein kinase signal pathway. Infect Immun. 2005;73(12):8209-8218.

96. Choi KS, Scorpio DG, Dumler JS. Anaplasma phagocytophilum ligation to toll-like receptor (TLR) 2, but not to TLR4, activates macrophages for nuclear factor- $\mathrm{\kappa B}$ nuclear translocation. J Infect Dis. 2004;189(10):1921-1925.

97. Scorpio DG, von Loewenich FD, Gobel H, Bogdan C, Dumler JS. Innate immune response to Anaplasma phagocytophilum contributes to hepatic injury. Clin Vaccine Immunol. 2006:13(7):806-809.

98. Muller BJ, Westheider A, Birkner K, Seelig B, Kirschnek S, Bogdan C, et al. Anaplasma phagocytophilum Induces TLRand MyD88-dependent signaling in in vitro generated murine neutrophils. Front Cell Infect Microbiol. 2021;11:627630.

99. Sukumaran B, Ogura Y, Pedra JH, Kobayashi KS, Flavell RA, Fikrig E. Receptor interacting protein-2 contributes to host defense against Anaplasma phagocytophilum infection. FEMS Immunol Med Microbiol. 2012;66(2):211-219.

100. Wang X, Shaw DK, Hammond HL, Sutterwala FS, Rayamajh M, Shirey KA, et al. The prostaglandin E2-EP3 receptor axis regulates Anaplasma phagocytophilum-mediated NLRC4 inflammasome activation. PLoS Pathog. 2016;12(8):e1005803. 
101. Naimi WA, Green RS, Cockburn CL, Carlyon JA. Correction: Naimi, W.A., et al. Differential susceptibility of male versus female laboratory mice to Anaplasma phagocytophilum Infection. Trop. Med. Infect. Dis. 2018, 3, 78. Trop Med Infect Dis. 2019;4(1):51

102. Bunnell JE, Trigiani ER, Srinivas SR, Dumler JS. Development and distribution of pathologic lesions are related to immune status and tissue deposition of human granulocytic ehrlichiosis agent-infected cells in a murine model system. J Infect Dis. 1999;180(2):546-550.

103. von Loewenich FD, Scorpio DG, Reischl U, Dumler JS, Bogdan C. Frontline: control of Anaplasma phagocytophilum, an obligate intracellular pathogen, in the absence of inducible nitric oxide synthase, phagocyte NADPH oxidase, tumor necrosis factor, Toll-like receptor (TLR)2 and TLR4, or the TLR adaptor molecule MyD88. Eur J Immunol. 2004;34(7):1789-1797.

104. Pedra JH, Sutterwala FS, Sukumaran B, Ogura Y, Qian F, Montgomery RR, et al. ASC/PYCARD and caspase-1 regulate the IL-18/IFN- $\delta$ axis during Anaplasma phagocytophilum infection. J Immunol. 2007;179(7):4783-4791.

105. Ewing SA, Dawson JE, Kocan AA, Barker RW, Warner CK, Panciera RJ, et al. Experimental transmission of Ehrlichia chaffeensis (Rickettsiales: Ehrlichieae) among white-tailed deer by Amblyomma americanum (Acari: Ixodidae). J Med Entomol. 1995;32(3):368-374.

106. Anderson BE, Dawson JE, Jones DC, Wilson KH. Ehrlichia chaffeensis, a new species associated with human ehrlichiosis. J Clin Microbiol. 1991;29(12):2838-2842.

107. Stone JH, Dierberg K, Aram G, Dumler JS. Human monocytic ehrlichiosis. J Am Med Assoc. 2004;292(18):2263-2270.

108. Lin M, Rikihisa Y. Ehrlichia chaffeensis and Anaplasma phagocytophilum lack genes for lipid A biosynthesis and incorporate cholesterol for their survival. Infect Immun. 2003;71(9):5324-5331.

109. Lin M, Rikihisa Y. Ehrlichia chaffeensis downregulates surface Toll-like receptors 2/4, CD14 and transcription factors PU. 1 and inhibits lipopolysaccharide activation of NF-KB, ERK 1/2 and p38 MAPK in host monocytes. Cell Microbiol. 2004;6(2):175-186.

110. Lina TT, Dunphy PS, Luo T, McBride JW. Ehrlichia Chaffeensis TRP120 activates canonical notch signaling to downregulate TLR2/4 expression and promote intracellular survival. mBio. 2016;7(4):e00672-e00716.

111. Miura K, Matsuo J, Rahman MA, Kumagai Y, Li X, Rikihisa $Y$. Ehrlichia chaffeensis induces monocyte inflammatory responses through MyD88, ERK, and NF-KB but not through TRIF, interleukin-1 receptor 1 (IL-1R1)/IL-18R1, or toll-like receptors. Infect Immun. 2011;79(12):4947-4956.

112. Koh YS, Koo JE, Biswas A, Kobayashi KS. MyD88-dependent signaling contributes to host defense against ehrlichial infection. PLoS One. 2010;5(7):e11758.

113. Chattoraj P, Yang Q, Khandai A, Al-Hendy O, Ismail N. TLR2 and Nod2 mediate resistance or susceptibility to fatal intracellular Ehrlichia infection in murine models of ehrlichiosis. PLoS One. 2013;8(3):e58514.

114. Kader M, Alaoui-El-Azher M, Vorhauer J, Kode BB, Wells JZ, Stolz D, et al. MyD88-dependent inflammasome activation and autophagy inhibition contributes to Ehrlichia-induced liver injury and toxic shock. PLoS Pathog. 2017;13(10):e1006644.

115. Wardrop NA, Thomas LF, Cook EA, de Glanville WA, Atkinson PM, Wamae CN, et al. The sero-epidemiology of Coxiella burnetii in humans and cattle, Western Kenya: evidence from a cross-sectional study. PLoS Negl Trop Dis. 2016;10(10):e0005032.

116. Georgiev M, Afonso A, Neubauer $\mathrm{H}$, Needham $\mathrm{H}$, Thiery $\mathrm{R}$, Rodolakis $\mathrm{A}$, et al. Q fever in humans and farm animals in four European countries, 1982 to 2010. Euro Surveill. 2013;18(8):pii=20407.
117. Alvarez-Alonso R, Basterretxea M, Barandika JF, Hurtado A, Idiazabal J, Jado I, et al. A Q fever outbreak with a high rate of abortions at a dairy goat farm: Coxiella burnetii shedding, environmental contamination, and viability. Appl Environ Microbiol. 2018;84(20):e01650-e01718.

118. Mioni MSR, Costa FB, Ribeiro BLD, Teixeira WSR, Pelicia VC, Labruna MB, et al. Coxiella burnetii in slaughterhouses in Brazil: a public health concern. PLoS One. 2020;15(10):e0241246.

119. Dobos A, Kreizinger Z, Kovacs AB, Gyuranecz M. Prevalence of Coxiella burnetii in Central and Eastern European dairy herds. Comp Immunol Microbiol Infect Dis. 2020;72:101489.

120. Million M, Raoult $D$. Recent advances in the study of $Q$ fever epidemiology, diagnosis and management. J Infect. 2015;71(Suppl 1):S2-S9.

121. Borawski K, Dunaj J, Pancewicz S, Krol M, Czupryna P, Moniuszko-Malinowska A. Coxiella burnetii and Q fever - a review. Przegl Epidemiol. 2020;74(1):43-48.

122. Budgin AM, Abidi MZ, Bajrovic V, Miller MA, Johnson SC. Severe acute $\mathrm{Q}$ fever pneumonia complicated by presumed persistent localized Q fever endocarditis in a renal transplant recipient: a case report and review of the literature. Transpl Infect Dis. 2020;22(1):e13230.

123. Leroy Q, Lebrigand K, Armougom F, Barbry P, Thiery R, Raoult D. Coxiella burnetii transcriptional analysis reveals serendipity clusters of regulation in intracellular bacteria. PLoS One. 2010;5(12):e15321.

124. Sandoz KM, Sturdevant DE, Hansen B, Heinzen RA. Developmental transitions of Coxiella burnetii grown in axenic media. J Microbiol Methods. 2014;96:104-110.

125. Omsland A, Cockrell DC, Howe D, Fischer ER, Virtaneva $K$, Sturdevant DE, et al. Host cell-free growth of the Q fever bacterium Coxiella burnetii. Proc Natl Acad Sci U S A. 2009;106(11):4430-4434.

126. van Schaik EJ, Chen C, Mertens K, Weber MM, Samuel JE. Molecular pathogenesis of the obligate intracellular bacterium Coxiella burnetii. Nat Rev Microbiol. 2013;11(8):561-573.

127. Pechstein J, Schulze-Luehrmann J, Bisle S, Cantet F, Beare PA, Olke M, et al. The Coxiella burnetii T4SS effector AnkF is important for intracellular replication. Front Cell Infect Microbiol. 2020;10:559915.

128. Morgan JK, Luedtke BE, Shaw El. Polar localization of the Coxiella burnetii type IVB secretion system. FEMS Microbiol Lett. 2010;305(2):177-183.

129. Moormeier DE, Sandoz KM, Beare PA, Sturdevant DE, Nair V, Cockrell DC, et al. Coxiella burnetii RpoS regulates genes involved in morphological differentiation and intracellular growth. J Bacteriol. 2019;201(8):e00009-e00019.

130. Cordsmeier A, Wagner N, Luhrmann A, Berens C. Defying death - how Coxiella burnetii copes with intentional host cell suicide. Yale J Biol Med. 2019;92(4):619-628.

131. Hackstadt T. Steric hindrance of antibody binding to surface proteins of Coxiella burnetti by phase I lipopolysaccharide. Infect Immun. 1988;56(4):802-807.

132. Moos A, Hackstadt T. Comparative virulence of intra- and interstrain lipopolysaccharide variants of Coxiella burnetii in the guinea pig model. Infect Immun. 1987;55(5):1144-1150.

133. Shannon JG, Howe D, Heinzen RA. Virulent Coxiella burnetii does not activate human dendritic cells: role of lipopolysaccharide as a shielding molecule. Proc Natl Acad Sci U S A. 2005;102(24):8722-8727.

134. Schoffelen T, Ammerdorffer A, Hagenaars JC, Bleeker-Rovers CP, Wegdam-Blans MC, Wever PC, et al. Genetic variation in pattern recognition receptors and adaptor proteins associated with development of chronic Q fever. J Infect Dis. 2015;212(5):818-829.

135. Ammerdorffer A, Schoffelen T, Gresnigt MS, Oosting M, den Brok MH, Abdollahi-Roodsaz S, et al. Recognition 
of Coxiella burnetii by toll-like receptors and nucleotidebinding oligomerization domain-like receptors. J Infect Dis. 2015;211(6):978-987.

136. Kohl L, Hayek I, Daniel C, Schulze-Luhrmann J, Bodendorfer B, Luhrmann A, et al. MyD88 is required for efficient control of Coxiella burnetii infection and dissemination. Front Immunol. 2019;10:165.

137. Bradley WP, Boyer MA, Nguyen HT, Birdwell LD, Yu J, Ribeiro $J M$, et al. Primary role for toll-like receptor-driven tumor necrosis factor rather than cytosolic immune detection in restricting Coxiella burnetii phase II replication within mouse macrophages. Infect Immun. 2016;84(4): 998-1015.

138. Zamboni DS, Campos MA, Torrecilhas AC, Kiss K, Samuel JE, Golenbock DT, et al. Stimulation of toll-like receptor 2 by Coxiella burnetii is required for macrophage production of pro-inflammatory cytokines and resistance to infection. J Biol Chem. 2004;279(52):54405-54415.

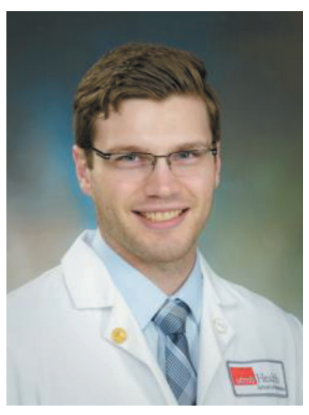

James Fisher received his Bachelor of Science in microbiology at the Pennsylvania State University, as well as his Master of Public Health from Yale University, with a concentration in epidemiology of microbial diseases. Currently, he is pursuing a combined $\mathrm{MD} / \mathrm{PhD}$ degree at the University of Texas Medical Branch, in Galveston, Texas, where he studies innate responses to Orientia tsutsugamushi. His research interests have focused on bacteriology, epidemiology, and immunology. 